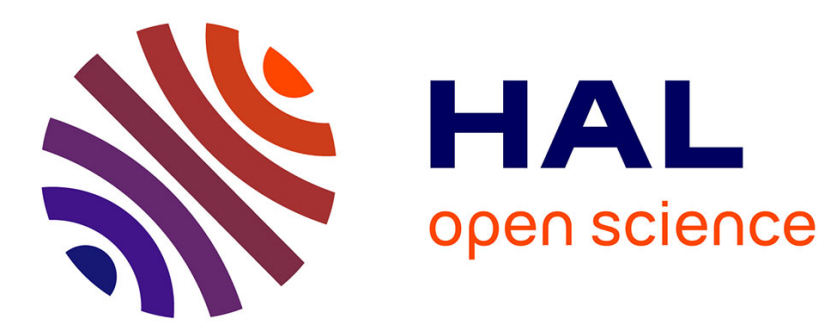

\title{
Hybrid proper orthogonal decomposition formulation for linear structural dynamics
}

\author{
Antoine Placzek, Duc-Minh Tran, Roger Ohayon
}

\section{To cite this version:}

Antoine Placzek, Duc-Minh Tran, Roger Ohayon. Hybrid proper orthogonal decomposition formulation for linear structural dynamics. Journal of Sound and Vibration, 2008, 318 (4-5), pp.943 - 964. 10.1016/j.jsv.2008.05.015 . hal-01517952

\section{HAL Id: hal-01517952 \\ https://hal.science/hal-01517952}

Submitted on 4 May 2017

HAL is a multi-disciplinary open access archive for the deposit and dissemination of scientific research documents, whether they are published or not. The documents may come from teaching and research institutions in France or abroad, or from public or private research centers.
L'archive ouverte pluridisciplinaire HAL, est destinée au dépôt et à la diffusion de documents scientifiques de niveau recherche, publiés ou non, émanant des établissements d'enseignement et de recherche français ou étrangers, des laboratoires publics ou privés.

\section{(c)(1)}

Distributed under a Creative Commons Attribution| 4.0 International License 


\title{
Hybrid proper orthogonal decomposition formulation for linear structural dynamics
}

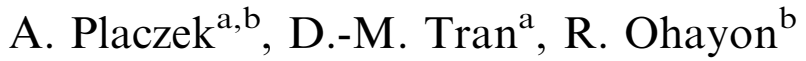 \\ ${ }^{a}$ ONERA, The French Aerospace Lab, Aeroelasticity and Structural Dynamics Department, \\ 29 avenue de la Division Leclerc, BP 72, 92320 Châtillon, France \\ ${ }^{\mathrm{b}}$ Conservatoire National des Arts et Métiers (CNAM), Structural Mechanics and Coupled Systems Laboratory, \\ 2 rue Conté, 75003 Paris, France
}

\begin{abstract}
Hybrid proper orthogonal decomposition (PODh) formulation is a POD-based reduced-order modeling method where the continuous equation of the physical system is projected on the POD modes obtained from a discrete model of the system. The aim of this paper is to evaluate the hybrid POD formulation and to compare it with other POD formulations on the simple case of a linear elastic rod subject to prescribed displacements in the perspective of building reduced-order models for coupled fluid-structure systems in the future. In the first part of the paper, the hybrid POD is compared to two other formulations for the response to an initial condition: an approach based on the discrete finite elements equation of the rod called the discrete POD (PODd), and an analytical approach using the exact solution of the problem and consequently called the analytical POD (PODa). This first step is useful to ensure that the PODh performs well with respect to the other formulations. The PODh is therefore used afterwards for the forced motion response where a displacement is imposed at the free end of the rod. The main contribution of this paper lies in the comparison of three techniques used to take into account the non-homogeneous Dirichlet boundary condition with the hybrid POD: the first method relies on control functions, the second on the penalty method and the third on Lagrange multipliers. Finally, the robustness of the hybrid POD is investigated on two examples involving firstly the introduction of structural damping and secondly a nonlinear force applied at the free end of the rod.
\end{abstract}

\section{Introduction}

The proper orthogonal decomposition (POD) is a powerful method providing the description of a highdimensional system by means of a small number of elements, called the POD modes (POMs). These POMs 
contain the main characteristics of the system response such that the solution of the system can be reconstructed as a linear combination of the POMs. The method is closely related to the Karhunen-Loève decomposition originating from the probability theory [1] and to the principal component analysis commonly used in the field of statistics. Connexions with the singular value decomposition can also be highlighted [2]. Liang et al. [3] demonstrated the equivalence between the preceding methods when they are used to handle discrete random vectors of finite dimension. According to Liang et al. [3], the POD method can thus be considered as a general term comprising the Karhunen-Loève decomposition, the principal component analysis and the singular value decomposition.

The starting point of the POD is a set of snapshots describing the system and obtained either from numerical simulations or experiments. The set of snapshots is combined into a correlation tensor, whose properties ensure that real eigenvectors exist. These eigenvectors are the POD modes and represent the coherent structures of the system. The most important feature of the POD is its optimality: for a given number of POMs, the POD basis containing these modes provides the best approximation of any element of the snapshots set. The POD is therefore a powerful technique for data analysis since an important database can be efficiently represented by only a small number of modes. Moreover, the interpretation of the database is simplified because the main characteristics of the system are contained within the POMs.

However, the method is not restricted to data analysis but can be combined with the equation governing a dynamical system to form a reduced-order model. Lumley [4] was one of the first to use POD in this context: using the POMs and drastic approximations to the Navier-Stokes equations, he derived a simplified model which was able to reproduce approximately the dynamics of flows characterized by large-scale spatially coherent structures. The Galerkin projection of the Navier-Stokes equations on the first POMs, namely the most energetic, leads to a low-dimensional system of nonlinear ordinary differential equations that can be solved very efficiently compared to direct numerical simulations. The POD method, which is originally only applicable to stationary stochastic fields, is justified for turbulent flows exhibiting a somewhat chaotic behavior, but could not a priori been used for a deterministic system. The method can, however, be formulated in a different way for such systems: the POD basis is sought as the solution of an optimization problem where the error in approximating any member of the snapshots set must be minimized. This leads to the same eigenproblem without adducing probabilistic arguments.

The POD has been extensively used for fluid dynamics systems because of the lack of efficient reduction methods for fluids. Indeed, the eigenmode reduction technique commonly used in structural dynamics is difficult to apply since the determination of fluid eigenmodes is a delicate issue. Fluids are generally governed by strongly nonlinear equations which have to be linearized before being able to define the eigenvalue problem from which the eigenmodes are obtained. The second limitation comes from the great number of degrees of freedom which in most cases prevents the extraction of the eigenmodes. These two limitations are avoided with the POD since (i) it can as well be formulated for a linear or a nonlinear system and (ii) the POMs can be obtained from the resolution of an eigenproblem whose dimension is equal to the number of snapshots and is consequently very small compared with the number of degrees of freedom. The construction of POD reducedorder models for the fluid has therefore expanded rapidly since the first works and a great part of the POD literature is devoted to fluid reduced-order models that could be built for a wide variety of flows to perform parametric studies or active control. Since the present paper focuses on a structural test case, the construction of POD reduced-order models for the fluid is not detailed here but the reader is referred to review articles like those of Lucia et al. [5] or Dowell et al. [6] for more details.

Historically, the POD-Galerkin method has been introduced for fluid dynamics systems but the technique has spread over various mechanical systems and notably structural mechanics ones. Kerschen et al. [7] give an overview of the general method and several examples of the use of POD (i) for the determination of nonlinear dynamical effects in continuous systems due to vibro-impact of a beam and a rotor, or (ii) for the dynamical analysis of linear truss dynamics where traditional modal analysis methods are difficult to apply as the modal densities are high. The POD method therefore provides an interesting alternative to the classical modal reduction using the modes of vibration, also called the eigenmodes in the following to contrast with the POMs. The POD is especially useful for nonlinear systems, since for linear systems and under additional assumptions, the POMs are closely related to the eigenmodes. Indeed, Feeny and Kappagantu [8] and Kerschen and Golinval [9] explained with two different approaches that the POMs converge to the eigenmodes of an 
undamped and unforced linear system when the mass matrix is proportional to identity and if a sufficient number of snapshots is considered. For such systems, the POD has no real advantage over the eigenmode reduction except if the modal densities are high. Thus POD is preferably applied to nonlinear systems [10,11], sometimes subject to vibro-impacts [12]. However, Sampaio and Soize [13] claim that the POD basis is not more efficient than the eigenmodes basis for nonlinear elastodynamics.

The construction of reduced-order models can be handled in different ways according to the nature (discrete or continuous) of the equation and snapshots used and the formulation often differs when fluids or solids are considered. For structural dynamics problems, the system is commonly discretized with finite elements; the POD eigenvalue problem is then constructed with the discretized snapshots and the resolution provides the generalized coordinates of the POMs. The matrix composed of the POMs is then directly used to project the discretized equation of the structure via a simple matricial product. This procedure is simple to implement and leads to a reduced-order model whose form is identical to the original discretized equation but with fewer degrees of freedom. This method will be referred in the following to the discrete POD (PODd). However, the whole discretized equation is not always available. Indeed, fluid mechanics problems, for example, involve a great number of degrees of freedom preventing the construction of the whole discretized matrices. As the reduced-order model cannot be directly formed from the discretized equation, the procedure consists in working from the continuous equations. The reduced-order model is then built by projecting the continuous equations on each POM according to the Galerkin method [14]. The difficulty is that POMs have been obtained with discrete snapshots resulting from the resolution of the equations discretized by a certain approximation formalism (finite differences, finite elements, finite volumes, etc.). The following step in the construction of the reduced-order model relies on the introduction of the discrete components of the POMs into the continuous formulation of the Galerkin projection. The most coherent approach consists in using the same formalism to connect the discrete components to the continuous formulation but other choices are possible. This formulation will be called the hybrid POD (PODh) since the discrete POMs are used in conjunction with the continuous equation.

Since our aim is to build later reduced-order models for fluids in the context of fluid-structure interaction simulations, we focus on the hybrid POD which is the common approach adopted for fluids. In this paper, the method is evaluated on the simple case of a free elastic rod subject to traction and compression. The use of POD for such a simple system has a very limited interest except for the evaluation of the hybrid POD formulation since an analytical solution can be exhibited. Although the application of the hybrid POD to a solid system seems quite paradoxical since we mentioned before that PODh is preferably used for fluids, this example is well-tailored for comparison purposes. Indeed, PODh as well as PODd can be easily formulated for the rod. Moreover, due to the extreme simplicity of the system, a third formulation based on the analytical solution of the system can be developed. This formulation, which is abbreviated in PODa, provides a reference to evaluate the errors relative to the two other formulations. It should be made clear that other reduction techniques (like the classical eigenmode reduction) are adapted to handle structural dynamics problems. However, our purpose is not to compare the hybrid POD to the eigenmode reduction method since this work has already been published in the literatures (see Ref. [13] for example). The eigenmode basis will only be used in the analytical POD formulation to define the exact solution of the system but it will not be used as a reduction method.

When dealing with fluid-structure interaction problems, the coupling condition between the fluid and the structure involves prescribed displacements on the fluid-structure interface. It is therefore of prime importance to investigate the forced response with prescribed displacements (non-homogeneous boundary condition) in the hybrid POD reduced-order model. Homogeneous boundary conditions are easily taken into account since those properties are inherited by the POMs. However, when non-homogeneous boundary conditions are applied to the system, the POD reduced-order model has to be adapted so that the boundary condition is satisfied. Three techniques are considered here to take into account a displacement imposed at the free end. The first method relies on a weak formulation of the boundary condition: the strong non-homogeneous Dirichlet boundary condition is transformed into a weak Neumann boundary condition by means of a small parameter whose inverse will be called the penalty factor. This method is therefore called the penalty method (PM). In the second approach, the system can be considered as a problem with a constraint which, in this case, represents the boundary condition that must be verified. The constraint is treated by means of a Lagrange 
multiplier, hence the name of Lagrange multiplier method (LMM) for the formulation. The last technique is based on a different formulation of the POD in which the POMs are computed for snapshots that have been previously homogenized. The homogenization step uses a suitably chosen "Control Function" that can be changed later in the reduced-order model to have the desired boundary condition. According to the paper of Tang et al. [15], this formulation will be called the control function method (CFM). The main contribution of this paper concerns the evaluation and the comparison of different techniques which can be used to take into account a prescribed displacement in the POD reduced-order model.

The paper is organized as follows: after having recalled the principle of the POD method in Section 2, the hybrid POD is compared with the PODd and the PODa in the case of the free response in Section 3. Thereafter, the different techniques (CFM, PM and LMM) developed for the hybrid POD to take into account the non-homogeneous boundary condition for the forced response are presented and evaluated in Section 4. Finally, in Section 5, two examples to investigate the robustness of the hybrid POD are performed.

\section{The proper orthogonal decomposition (POD) method}

The POD method is presented here in the context of a deterministic system and we do not refer to the Karhunen-Loève theorem usually adduced in the stochastic setting. The description, close to the one of Holmes et al. [16], relies on an optimization problem which consists in finding the best basis to approximate a set of snapshots. The snapshots are in fact solutions $u^{(k)}$ of the system evaluated at different instants $t_{k}$ and the snapshots set $U=\left\{u^{(k)} \in H, k=1, \ldots, M\right\}$ gathers a finite number of them. The solutions are contained in a Hilbert space $H$ with $N=\operatorname{dim}(H)$ ( $N$ being eventually infinite), whose vectors can be continuous squared integrable functions on a spatial domain $\Omega\left(H=L^{2}(\Omega)\right)$ or real discrete finite vectors $\left(H=\mathbb{R}^{N}\right)$ for example. Let $\langle\cdot, \cdot\rangle$ be the inner product associated to the Hilbert space $H$ and $\|\cdot\|$ denote the norm induced by this inner product so that $\forall x \in H,\|x\|^{2}=\langle x, x\rangle$.

The POD consists then in finding a subspace $S \subset H$ of finite dimension $q \ll N$ which provides the best approximation of any member of $U$. This subspace is entirely characterized by the basis $\left\{\varphi^{(j)} \in H, j=1, \ldots, q\right\}$ so that $S=\operatorname{span}\left\{\varphi^{(1)}, \ldots, \varphi^{(q)}\right\}$. Each snapshot can therefore be approximated on the subspace $S$ by a linear combination of the POMs $\varphi^{(j)}$ :

$$
u^{(k)}=\sum_{j=1}^{q} a_{j}^{(k)} \varphi^{(j)} \quad \forall k \in[1 ; M],
$$

where the $a_{j}^{(k)}$ are real scalars representing the modal amplitudes associated to the mode $\varphi^{(j)}$ in the decomposition of the snapshot $u^{(k)}$. The subspace $S$ has to be sought so that the basis $\left\{\varphi^{(j)}\right\}_{j=1}^{q}$ is optimal in the sense that it is better than any other basis of same dimension $q$. To ensure optimality, we impose that the timeaveraged squared projection error $E\left(\left\|u^{(k)}-\mathscr{P}_{S} u^{(k)}\right\|^{2}\right)$ is minimal. The time-averaging operation for a function $f\left(u^{(k)}\right)$ depending on the snapshots is the sum $E\left(f\left(u^{(k)}\right)\right)=\sum_{k=1}^{M} \alpha_{k} f\left(u^{(k)}\right)$ where the $\alpha_{k}$ are weighting coefficients. The minimization problem is equivalent to maximizing the time-averaged squared projection of the snapshots $E\left(\left\|\mathscr{P}_{S} u^{(k)}\right\|^{2}\right)$. Indeed the operator $\mathscr{P}_{S}$ defines an orthogonal projection on the subspace $S$ in order to minimize the distance between each snapshot in $H$ and its approximation in $S$. Developing the expression of the orthogonal projector in $\mathscr{P}_{S} u^{(k)}=\sum_{j=1}^{q}\left\langle u^{(k)}, \varphi^{(j)}\right\rangle \varphi^{(j)}$ and using the orthonormality of the POMs, the mathematical statement of optimality is to find the

$$
\max _{\varphi^{(j)} \in H,\left\|\varphi^{(j)}\right\|=1} \sum_{j=1}^{q} E\left(\left\langle u^{(k)}, \varphi^{(j)}\right\rangle^{2}\right)
$$

for suitably normalized POMs $\varphi^{(j)}$. The problem of finding the maximum subject to the constraint $\left\|\varphi^{(j)}\right\|=1$ can be transformed into the following variational problem defined for the functional $J$ :

$$
J\left[\varphi^{(j)}\right]=E\left(\left\langle u^{(k)}, \varphi^{(j)}\right\rangle^{2}\right)-\lambda_{j}\left(\left\|\varphi^{(j)}\right\|^{2}-1\right)
$$

A necessary condition to obtain the maximum is that the functional derivative vanishes for all variations $\varphi^{(j)}+\delta \psi \in H, \delta \in \mathbb{R}$. After some mathematical manipulations (for more details, see Ref. [16]), the preceding condition which must be true for all variation $\psi$, turns out to be equivalent to solving the following eigenvalue 
problem:

$$
R \varphi^{(j)}=\lambda_{j} \varphi^{(j)} \quad \forall j \in[1 ; q]
$$

where the operator $R$ is an endomorphism of $H$ defined by

$$
R=E\left(u^{(k)} \otimes\left(u^{(k)}\right)^{*}\right)
$$

In the preceding expression, $\left(u^{(k)}\right)^{*}$ is the dual of the vector $u^{(k)}$, i.e. the linear application defined by $\left(u^{(k)}\right)^{*}: v \longrightarrow\left(u^{(k)}\right)^{*} v=\left\langle v, u^{(k)}\right\rangle, \forall v \in H$ and $\otimes$ is the usual tensor product so that $w \otimes\left(u^{(k)}\right)^{*} v=$ $w\left\langle v, u^{(k)}\right\rangle, \forall v, w \in H$. The operator $R$ is self-adjoint and has a finite rank $r$ since it is spanned by the snapshots. $R$ is therefore a Hilbert-Schmidt operator and is consequently compact. With these properties the spectral theory for compact self-adjoint operators is valid and ensures that there is only a finite number $r$ of real nonnull eigenvalues $\lambda_{j}$. The associated eigenvectors which are the POMs we looked for are orthonormal.

Taking the inner product of Eq. (4) with $\varphi^{(j)}$ and using the expression Eq. (5) of the operator $R$ lead to the following relation:

$$
\lambda_{j}=E\left(\left\langle u^{(k)}, \varphi^{(j)}\right\rangle^{2}\right)
$$

The preceding relation indicates that the greatest eigenvalues $\lambda_{j}$ maximize in average the projection of the snapshots on the POMs. Consequently, the greatest eigenvalues capture the most of "energy" contained in the snapshots set in the sense of the norm induced by the Hilbert space. In order to be efficient, the reduced-order model constructed with the POMs has therefore to be composed of the first $q \leqslant r$ eigenvectors associated to the largest eigenvalues.

The process described previously would face the same problem as the one mentioned in the introduction for the eigenmode extraction (for classical modal reduction) when the size of the problem becomes important. Indeed, the dimension of the operator $R$ is related to the number of degrees of freedom in the system. A powerful variant called the snapshots method proposed by Sirovich [17] reduces considerably the size of the problem to be solved. The method is based on the fact that the POMs are a linear combination of the snapshots:

$$
\varphi^{(j)}=\sum_{k=1}^{M} c_{k}^{(j)} u^{(k)}
$$

since the image of $R$ is a subset of the space spanned by the snapshots. Introducing Eq. (7) into Eq. (4) with the expression Eq. (5) of $R$ leads, after reorganization of the terms, to

$$
\left.\sum_{k=1}^{M} \sum_{l=1}^{M} \alpha_{k}\left\langle u^{(k)}, u^{(l)}\right\rangle c_{l}^{(j)}\right) u^{(k)}=\lambda_{j} \sum_{m=1}^{M} c_{m}^{(j)} u^{(m)}
$$

If the snapshots are assumed to be linearly independent, the previous expression reduces to the new eigenvalue problem whose dimension is now $M \ll N$ :

$$
\sum_{l=1}^{M} \alpha_{k}\left\langle u^{(k)}, u^{(l)}\right\rangle c_{l}^{(j)}=\lambda_{j} c_{k}^{(j)} \quad \forall k \in[1 ; M]
$$

The previous relation can be transposed in the matricial form $\widetilde{\mathbf{R}}^{*} \mathbf{c}^{(j)}=\lambda_{j} \mathbf{c}^{(j)}$ with $\widetilde{R}_{i j}^{*}=\alpha_{i}\left\langle u^{(i)}, u^{(j)}\right\rangle$ and $\mathbf{c}^{(j)}=\left[c_{1}^{(j)} \cdots c_{M}^{(j)}\right]^{\mathrm{T}}$. However, the matrix $\widetilde{\mathbf{R}}^{*}$ is not symmetrical except if all the weights $\alpha_{i}$ associated to the time-averaging operator $E(\cdot)$ are identical. If all weights are strictly positive, the problem can be symmetrized by using the matrix $\widetilde{\alpha}=\operatorname{diag}\left(\sqrt{\alpha_{1}}, \ldots, \sqrt{\alpha_{M}}\right)$. The eigenvalue problem obtained with the snapshots method is finally:

$$
\mathbf{R}^{*} \mathbf{d}^{(j)}=\lambda_{j} \mathbf{d}^{(j)}
$$

where $R_{i j}^{*}=\sqrt{\alpha_{i} \alpha_{j}}\left\langle u^{(i)}, u^{(j)}\right\rangle$ and the eigenvectors are related to the coefficients $\mathbf{c}^{(j)}$ of the decomposition Eq. (7) by the following relation $\mathbf{c}^{(j)}=\widetilde{\boldsymbol{\alpha}} \mathbf{d}^{(j)}$.

The snapshots method has been obtained here for snapshots of possibly infinite dimension. The equivalence with the direct method holds only if the snapshots are linearly independent, so that one can infer Eq. (9) from 
Eq. (8). If snapshots of finite dimension are considered, it is not necessary to assume that they are linearly independent. The equivalence between the direct method and the snapshots method can be proved for example by using the singular value decomposition of the matrix containing the snapshots of finite dimension. In this case, the snapshots method provides as many POMs as the rank $r$ of the snapshots matrix and the POMs are strictly identical to those of the direct method. For practical applications, finite dimension snapshots are always used and consequently the snapshots method will be preferably adopted in the remainder of this paper.

It should also be noted that better results are obtained when the POD is performed for the fluctuations $s^{(k)}$ of the data rather than for the raw data $u^{(k)}$. Indeed, Tamura et al. [18] observed that if the POD is carried out directly for the snapshots $u^{(k)}$, the first POD mode is rather similar to the mean of the snapshots and the eigenspectra is distorted. The fluctuations, or centered data, are defined by $s^{(k)}=u^{(k)}-\bar{u}$, where $\bar{u}=E\left(u^{(k)}\right)$ represents the time-average of the snapshots. The operators $R$ and $R^{*}$ are thus based on the $s^{(k)}$ instead of the $u^{(k)}$ and the decomposition Eq. (1) of the snapshots is modified in:

$$
u^{(k)}=\bar{u}+\sum_{j=1}^{q} a_{j}^{(k)} \varphi^{(j)} \quad \forall k \in[1 ; M]
$$

\section{Hybrid POD formulation for the response to an initial condition}

We present here the hybrid POD formulation (PODh) in the case of the free response. The PODh will be evaluated and compared to two other formulations, namely the discrete formulation (PODd) and the analytical formulation (PODa), which are briefly described in the following. The problem considered is a one-dimensional rod subject to traction and compression. The rod is characterized by its length $L$, the section $S$ and the material is represented by the Young's modulus $E$ and the density $\rho$. In absence of external forces, the displacement $u(x, t)$ of the rod section at a given point $x$ and at time $t$ is governed by the wave equation:

$$
\rho S \frac{\partial^{2} u}{\partial t^{2}}-E S \frac{\partial^{2} u}{\partial x^{2}}=0
$$

A unique solution is specified with a set of boundary conditions corresponding to a clamped-free rod and initial conditions representing an initial displacement $U_{0}$ applied at the free end:

$$
\left\{\begin{array}{l}
u(x=0, t)=0 \\
\left.E S \frac{\partial u}{\partial x}\right|_{x=L}=0
\end{array} \quad \forall t>0, \quad\left\{\begin{array}{l}
u(x, t=0)=\left(U_{0} / L\right) x \\
\left.\frac{\partial u}{\partial t}\right|_{t=0}=0
\end{array} \quad \forall x \in[0 ; L]\right.\right.
$$

The following paragraphs present the different reduced-order models built from the preceding equation according to the approach considered.

\subsection{Hybrid POD formulation (PODh)}

The aim of this formulation is to use a discrete snapshots database in conjunction with the continuous equation of the system. Indeed, the snapshots are almost always described by a discrete quantity coming either from a numerical simulation or an experiment. Nonetheless, the discrete equation that could be combined with this discrete approximation of the snapshots is not always available and the continuous equation will therefore be necessary. If the snapshots have been obtained from numerical simulations, the most coherent approach consists in using the same approximation formalism to link the discrete variable and the continuous equation. However, it is not always possible to use the same formalism and sometimes it is even not available (this is the case for example when the snapshots stem from experiments).

The dataset available is used to approximate directly the $L^{2}$ inner product which appears in the PODh formulation since it relies on the continuous equation of the system. The inner product of two continuous 
functions $f$ and $g$ is therefore approximated by the trapezoidal rule:

$$
\langle f, g\rangle_{L^{2}(\Omega)}=\int_{\Omega} f(x) g(x) \mathrm{d} x=\sum_{e=1}^{N} \int_{\Omega_{e}} f(x) g(x) \mathrm{d} x \approx \sum_{e=1}^{N} \widehat{f}_{e} \widehat{g}_{e} \delta \Omega_{e}
$$

where $\widehat{f}_{e}$ and $\widehat{g}_{e}$ are the average values relative to the functions $f$ and $g$ evaluated on each element $e$ and $\delta \Omega_{e}$ is the Lebesgue measure of the element $\Omega_{e}$. The previous expression takes the matricial form: $\langle f, g\rangle_{L^{2}(\Omega)} \approx \widehat{\mathbf{f}}^{\mathrm{T}} \Delta \widehat{\mathbf{g}}$ with $\widehat{\mathbf{f}}=\left[\widehat{f}_{1} \ldots \widehat{f}_{N}\right]^{\mathrm{T}}$ (resp., for $\widehat{\mathbf{g}}$ ) and $\boldsymbol{\Delta}=\operatorname{diag}\left(\delta \Omega_{1}, \ldots, \delta \Omega_{N}\right)$. As the problem is unidimensional, the average values of the function $f$ on one element is simply given here by the arithmetic mean $\widehat{f}_{i}=\left(f_{i}+f_{i+1}\right) / 2$, where the $f_{i}$ are the values contained in the dataset and which are supposed to be the values at the nodes of the mesh.

The correlation tensor $\mathbf{R}^{*}$ is defined by the $L^{2}$ inner product since the reduced-order model is built from the continuous equation. The inner product is then approximated with Eq. (14) so that the general term of $\mathbf{R}^{*}$ is: $R_{i j}^{*}=\sqrt{\alpha_{i} \alpha_{j}}\left\langle s^{(i)}, s^{(j)}\right\rangle_{L^{2}} \approx \sqrt{\alpha_{i} \alpha_{j}}\left(\widehat{\mathbf{s}}^{(i)}\right)^{\mathrm{T}} \Delta \widehat{\mathbf{s}}^{(j)}$. With matricial notations, the previous relation is rewritten as $\mathbf{R}^{*}=\left(\widetilde{\boldsymbol{\alpha}}^{\mathrm{T}} \widehat{\mathbf{S}}^{\mathrm{T}}\right) \boldsymbol{\Delta}(\widehat{\mathbf{S}} \widetilde{\boldsymbol{\alpha}})$. The POMs extracted from the eigenvalue problem are discrete like the snapshots they have been derived from. In order to build the reduced-order model, the continuous POD decomposition Eq. (11) is substituted into the governing equation (12) and the resulting expression is projected onto each POM:

$$
\rho S \sum_{k=1}^{q} \frac{\mathrm{d}^{2} a_{k}}{\mathrm{~d} t^{2}}\left\langle\varphi^{(k)}, \varphi^{(j)}\right\rangle=E S \sum_{k=1}^{q} a_{k}\left\langle\frac{\mathrm{d}^{2} \varphi^{(k)}}{\mathrm{d} x^{2}}, \varphi^{(j)}\right\rangle+E S\left\langle\frac{\mathrm{d}^{2} \bar{u}}{\mathrm{~d} x^{2}}, \varphi^{(j)}\right\rangle
$$

The integrals involved in the $L^{2}$ inner product allow parts integrations to be performed so that the order of the derivatives can be lowered. In this case, the boundary terms vanish since the POMs, which are linear combinations of the snapshots, inherits the homogeneous boundary conditions defined in Eq. (13). Finally, the left-hand side term of Eq. (15) can be simplified due to the orthonormality of the POMs and the equation governing the modal amplitudes writes:

$$
\rho S \frac{\mathrm{d}^{2} a_{j}}{\mathrm{~d} t^{2}}=-E S \sum_{k=1}^{q}\left\langle\frac{\mathrm{d} \varphi^{(k)}}{\mathrm{d} x}, \frac{\mathrm{d} \varphi^{(j)}}{\mathrm{d} x}\right\rangle a_{k}-E S\left\langle\frac{\mathrm{d} \bar{u}}{\mathrm{~d} x}, \frac{\mathrm{d} \varphi^{(j)}}{\mathrm{d} x}\right\rangle
$$

The reduced-order model is not usable under this continuous form since only the discrete forms of the POMs and snapshots are known. Firstly, the inner products are approximated according to Eq. (14). The first inner product writes $\left(\widehat{d \varphi^{(k)}}\right)^{\mathrm{T}} \boldsymbol{\Delta}\left(\widehat{d \varphi^{(j)}}\right)$ and the second is $(\widehat{d \overline{\mathbf{u}}})^{\mathrm{T}} \boldsymbol{\Delta}\left(\widehat{d \varphi^{(j)}}\right)$, where $d$ is a notation standing for the spatial derivative: $d \varphi^{(i)}$ is thus the discrete approximation corresponding to the derivative of the discrete vector $\varphi^{(i)}$ and similarly $d \overline{\mathbf{u}}$ is the derivative of the time-averaged part. The second and last step to obtain the reducedorder model consists in computing the derivatives of the POMs and of the time-averaged part with a numerical approximation scheme based for example on finite differences (a central differences scheme will be adopted here because of its second-order accuracy). The mean values of the derivative vectors $d \varphi^{(i)}$ and $d \overline{\mathbf{u}}$ are then computed so that the matrix $\widehat{d \boldsymbol{\Phi}}=\left[\widehat{d \varphi^{(1)}} \ldots \widehat{d \varphi^{(q)}}\right]$ and the vector $\widehat{d \overline{\mathbf{u}}}$ can be constructed.

The $q$ equations obtained by projecting on each POM can be gathered in the matricial ordinary differential equation $\ddot{\mathbf{a}}=\mathscr{L} \mathbf{a}+\mathscr{K}$ defining the unknown amplitudes $\mathbf{a}=\left[a_{1} \ldots a_{q}\right]^{\mathrm{T}}$ of the decomposition Eq. (11). Denoting $c=\sqrt{E / \rho}$, the matrix $\mathscr{L}$ and the vector $\mathscr{K}$ are given by

$$
\mathscr{L}=-c^{2}(\widehat{d \boldsymbol{\Phi}})^{\mathrm{T}} \boldsymbol{\Delta}(\widehat{d \boldsymbol{\Phi}}), \quad \mathscr{K}=-c^{2}(\widehat{d \boldsymbol{\Phi}})^{\mathrm{T}} \boldsymbol{\Delta} \widehat{d \overline{\mathbf{u}}}
$$

\subsection{Discrete POD formulation (PODd)}

Instead of using a continuous framework, the equation governing the rod can be discretized with the finite elements method. The continuous solution $u(x, t)$ is approximated by a finite number of generalized coordinates $u_{j}$ such that $u(x, t) \approx \sum_{j=1}^{N} u_{j}(t) \chi_{j}(x)$ where $N$ is the number of nodes used for the discretization and the $\chi_{j}(x)$ are linear shape functions. The weak variational formulation of Eq. (12) leads to the following matricial equation: $\mathbf{M} \ddot{\mathbf{u}}+\mathbf{K u}=\mathbf{0}$, where $\mathbf{u}=\left[u_{1} \cdots u_{N}\right]^{\mathrm{T}}$ is the vector containing the unknown generalized coordinates and $\mathbf{M}$ and $\mathbf{K}$ are, respectively, the mass and rigidity matrices. 
The continuous variable $u$ is now entirely represented by its generalized coordinates $\mathbf{u}$ and the snapshots will be considered under this discrete form rather than the continuous one. We, therefore, introduce the matrix of the snapshots $\mathbf{U}=\left[\mathbf{u}^{(1)} \cdots \mathbf{u}^{(M)}\right]$ and the centered matrix of the snapshots $\mathbf{S}=\left[\mathbf{s}^{(1)} \cdots \mathbf{s}^{(M)}\right]=\mathbf{U}-\overline{\mathbf{U}}$. The matrix $\overline{\mathbf{U}}$ contains the time-averaged part of the snapshots which is subtracted from each component such that $S_{i j}=s_{i}^{(j)}=u_{i}^{(j)}-E\left(u_{i}^{(k)}\right)$. In opposition to the hybrid formulation, the operator $\mathbf{R}^{*}$ is now defined for the discrete Hilbert space $H=\mathbb{R}^{N}$ of finite dimension. In this case, the components of the correlation tensor $\mathbf{R}^{*}$ are obtained with the canonical inner product of $\mathbb{R}^{N}: R_{i j}^{*}=\sqrt{\alpha_{i} \alpha_{j}}\left\langle\mathbf{s}^{(i)}, \mathbf{s}^{(j)}\right\rangle_{\mathbb{R}^{N}}=\sqrt{\alpha_{i} \alpha_{j}} \sum_{l=1}^{N} s_{l}^{(i)} s_{l}^{(j)}$ and the inner product can be interpreted as the following matricial product: $\mathbf{R}^{*}=\widetilde{\alpha}^{\mathrm{T}} \mathbf{S}^{\mathrm{T}} \mathbf{S} \widetilde{\boldsymbol{\alpha}}$. The resolution of Eq. (10) with the new expression of $\mathbf{R}^{*}$ provides the coefficients $\mathbf{d}^{(j)}$ and $\mathbf{c}^{(j)}$ that define the POMs by Eq. (7). However, attention must be paid to the fact that the POMs are now a linear combination of discrete snapshots $\mathbf{u}$ since the Hilbert space is $\mathbb{R}^{N}$. For the sake of clarity, the relation is written $\varphi^{(j)}=\sum_{k=1}^{M} c_{k}^{(j)} \mathbf{s}^{(k)}$ or with matricial notations $\boldsymbol{\Phi}=\mathbf{S C}$, where $\mathbf{C}=\left[\mathbf{c}^{(1)} \ldots \mathbf{c}^{(q)}\right]$ and $\boldsymbol{\Phi}=\left[\boldsymbol{\varphi}^{(1)} \ldots \varphi^{(q)}\right]$ is the matrix containing the generalized coordinates of the POMs. The vector of the generalized coordinates $\mathbf{u}$ is thus approximated by $\mathbf{u}=\overline{\mathbf{u}}+\boldsymbol{\Phi a}$, which is the discrete form of Eq. (11). The reduced-order model is then built directly from the discrete equation by introducing the previous decomposition into $\mathbf{M} \ddot{\mathbf{u}}+\mathbf{K u}=\mathbf{0}$ and projecting on the POMs matrix $\boldsymbol{\Phi}$. This results in: $\mathbf{M}_{q} \ddot{\mathbf{a}}+\mathbf{K}_{q} \mathbf{a}=\mathbf{F}_{q}$, with $\mathbf{M}_{q}=\boldsymbol{\Phi}^{\mathrm{T}} \mathbf{M \Phi}, \mathbf{K}_{q}=\boldsymbol{\Phi}^{\mathrm{T}} \mathbf{K} \boldsymbol{\Phi}$ and $\mathbf{F}_{q}=-\boldsymbol{\Phi}^{\mathrm{T}} \mathbf{K} \overline{\mathbf{u}}$. Since the matrix $\mathbf{M}_{q}$ is symmetric and positive definite, the preceding equation can be pre-multiplied by $\mathbf{M}_{q}^{-1}$ to regain the same form of the reduced-order model as for the hybrid formulation. The matrix $\mathscr{L}$ and the vector $\mathscr{K}$ are now defined by

$$
\mathscr{L}=-\mathbf{M}_{q}^{-1} \mathbf{K}_{q}, \quad \mathscr{K}=-\mathbf{M}_{q}^{-1} \mathbf{F}_{q}
$$

\subsection{Analytical POD formulation (PODa)}

For the free response, an analytical expression can be easily exhibited by assuming the separation of time and space for the solution which can be written $u(x, t)=\sum_{n=1}^{\infty} \zeta_{n} \Theta_{n}(t) \psi_{n}(x)$, with $\zeta_{n}=(-1)^{n-1}\left(4 \sqrt{2 L} U_{0}\right) /$ $((2 n-1) \pi)^{2}, \Theta(t)=\cos \left(k_{n} t\right)$ and $\psi_{n}(x)=\sqrt{2 / L} \sin \left(\left(k_{n} x\right) / c\right)$. The constants $k_{n}=(2 n-1) \pi c /(2 L), \forall n \in \mathbb{N}^{*}$ are the wavenumbers stemming from the boundary conditions and the parameter $c=\sqrt{E / \rho}$ represents the speed of sound propagation in the rod. The fundamental period of the system which will be used later for the adimensionalization of time, is $T_{0}=4 L / c$.

The analytical solution involves series of trigonometric functions, which, in this particular case, coincide with the eigenmodes of the clamped-free rod. The spatial functions $\psi_{n}(x)$ are thus the eigenmodes which are usually obtained from the generalized eigenvalue problem associated to the conservative part of the elastic system. Although it is here talked of the eigenmode basis, it does not be understood as a reduction method but rather as the analytical solution of the system. This basis will only serve to formulate the analytical POD and to evaluate later the POMs by comparison with the eigenmodes.

The analytical expression of the displacements can be used to obtain the expression of the snapshots, which can be decomposed on the eigenmode basis with the coefficients $\zeta_{n}^{(k)}=\zeta_{n} \cos \left(k_{n} t_{k}\right)$ :

$$
u^{(k)}(x)=\sum_{n=1}^{\infty} \zeta_{n}^{(k)} \psi_{n}(x)
$$

Eq. (19) provides a continuous expression of the snapshots which can be considered as vectors of the Hilbert space $H=L^{2}(\Omega)$ with $\Omega=[0 ; L]$. The POMs are obtained with the snapshots method for which the operator $\mathbf{R}^{*}$ is defined on $H=L^{2}$. The general term $R_{i j}^{*}=\sqrt{\alpha_{i} \alpha_{j}}\left\langle s^{(i)}, s^{(j)}\right\rangle_{L^{2}}$ can be simplified since the eigenmodes $\psi_{n}$ are orthonormal functions for the $L^{2}$ inner product. This leads to $R_{i j}^{*}=\sqrt{\alpha_{i} \alpha_{j}} \sum_{n=1}^{\infty}\left(\zeta_{n}^{(i)}-\overline{\zeta_{n}}\right)\left(\zeta_{n}^{(j)}-\overline{\zeta_{n}}\right)$, where $\bar{\zeta}_{n}=E\left(\zeta_{n}^{(k)}\right)$ is the time-averaged part of the amplitudes $\zeta_{n}^{(k)}$. The eigenvalue problem Eq. (10) is then solved with the previous expression of $\mathbf{R}^{*}$ to find the eigenvectors $\mathbf{d}^{(j)}$ and $\mathbf{c}^{(j)}=\widetilde{\alpha} \mathbf{d}^{(j)}$ so that the POMs are known with Eq. (7). As the POMs are linear combinations of the snapshots, they can be decomposed on the 
eigenmode basis $\left\{\psi_{n}\right\}_{n \geqslant 1}$ which spans the space of the solutions containing the snapshots:

$$
\varphi^{(j)}(x)=\sum_{n=1}^{\infty} \mu_{n}^{(j)} \psi_{n}(x) \quad \text { with } \mu_{n}^{(j)}=\sum_{l=1}^{M}\left(\zeta_{n}^{(l)}-\bar{\zeta}_{n}\right) c_{l}^{(j)}
$$

The reduced-order model associated to the analytical expression of the snapshots is obtained by first introducing the decomposition Eq. (11) of the variable into Eq. (12) governing the dynamics of the system. If the decomposition Eq. (19) of the snapshots on the eigenmode basis is used (and similarly for the decomposition Eq. (20) of the POMs), analytical expressions of the spatial derivatives can be obtained, so that Eq. (12) becomes

$$
\left.\rho S \sum_{k=1}^{q} \frac{\mathrm{d}^{2} a_{k}}{\mathrm{~d} t^{2}} \varphi^{(k)}+E S \sum_{k=1}^{q} \sum_{n=1}^{\infty}\left(\frac{k_{n}}{c}\right)^{2} \mu_{n}^{(k)} \psi_{n}\right) a_{k}+E S \sum_{n=1}^{\infty}\left(\frac{k_{n}}{c}\right)^{2} \bar{\zeta}_{n} \psi_{n}=0
$$

Finally, the reduced-order model is obtained by projecting the preceding equation on each POM $\varphi^{(j)}$ according to the Galerkin projection method. The first term with the time derivative reduces to $\rho S \ddot{a}_{j}$ since the POMs are orthonormal. In the two remaining terms, the inner product between a POM $\varphi^{(j)}$ and an eigenmode $\psi_{n}$ reduces to $\left\langle\psi_{n}, \varphi^{(j)}\right\rangle=\mu_{n}^{(j)}$. The reduced-order model developed with this last formulation keeps the same structure as the two preceding but the components of the matrix $\mathscr{L}$ and of the vector $\mathscr{K}$ are now given by:

$$
\mathscr{L}_{i j}=-\sum_{n=1}^{\infty} k_{n}^{2} \mu_{n}^{(i)} \mu_{n}^{(j)}, \quad \mathscr{K}_{i}=-\sum_{n=1}^{\infty} k_{n}^{2 \bar{\zeta}_{n}} \mu_{n}^{(i)}
$$

The analytical expressions of the coefficients involve infinite series that have to be truncated for numerical applications. Consequently, only a finite number $p$ of eigenmodes is used to evaluate the coefficients of the reduced-order model.

\subsection{Hybrid POD evaluation for the response to an initial condition}

In this section, the hybrid POD formulation is evaluated in the case of the free response. The time integration of the reduced-order model is easily performed by the ODEPACK solver. The efficiency of the

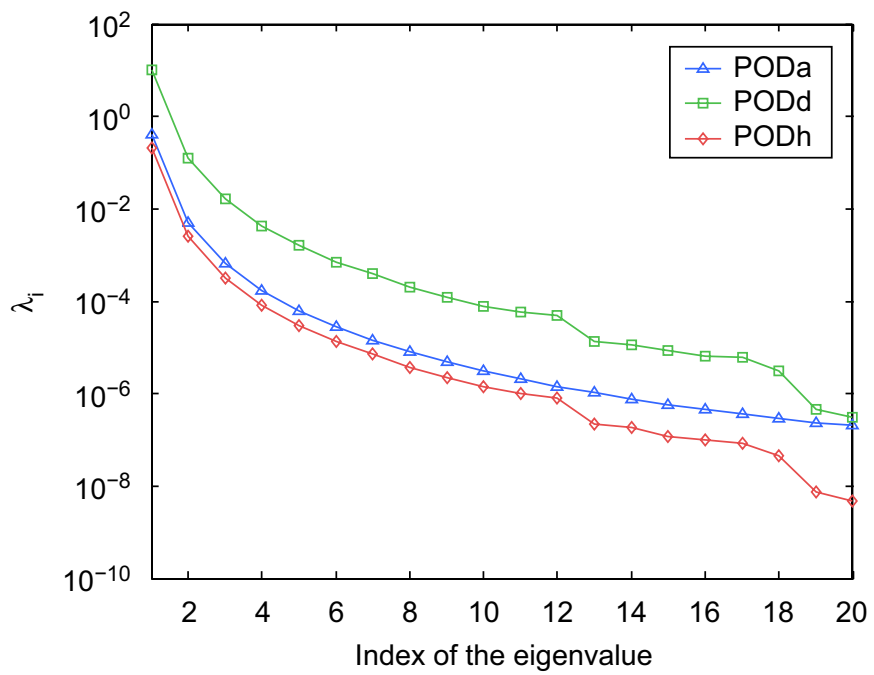

Fig. 1. Comparison of the eigenvalue spectra relative to the correlation tensor $\mathbf{R}^{*}$ of each POD formulation. 
$\mathrm{ROM}$ is evaluated by means of the following spatio-temporal error $\varepsilon_{\infty}$ defined for the discrete responses:

$$
\varepsilon_{\infty}=\frac{\left\|\mathbf{U}-\mathbf{U}_{r}\right\|_{\infty}}{\left\|\mathbf{U}_{r}\right\|_{\infty}}
$$

The matrix $\mathbf{U}$ contains the response obtained with the POD reduced-order model at each point of the mesh and at each discrete instant depending on the time step used for the time integration of the reduced-order model. Similarly, the matrix $\mathbf{U}_{r}$ is the reference solution evaluated at the same points in space and time.

The hybrid formulation of the POD is briefly compared here to the discrete and analytical formulations. The analytical formulation uses $p=N$ eigenmodes, i.e. as many as the number of elements used for the discretized approach. Fig. 1 presents the POMs eigenspectra relative to each POD formulation. The spectrum corresponding to the analytical formulation is clearly much more regular than the two others since the PODa uses the analytical solution of the snapshots Eq. (19) to form the correlation tensor, whereas the other formulations (PODd and PODh) rely on discrete snapshots stemming from a numerical finite elements simulation.

However, the eigenspectra have globally the same shape except small irregularities about the 12th and 18th eigenvalues. The most important point is that the slopes of the eigenspectra are nearly identical at least for the first eigenvalues, so that in each case the first five eigenvalues that will be kept in the ROM contain $99.98 \%$ of the "energy" of the system. The percentage of "energy" captured by the reduced-order model is defined by the ratio $\sum_{j=1}^{q} \lambda_{j} / \sum_{j=1}^{r} \lambda_{j}$ since the eigenvalues represent the "energy" of the system captured by each POM, see Eq. (6).

The POMs computed with the three formulations are very similar and the differences are barely distinguishable on the first POMs (see Fig. 2). The POMs computed with the snapshots of the free response are

(a)

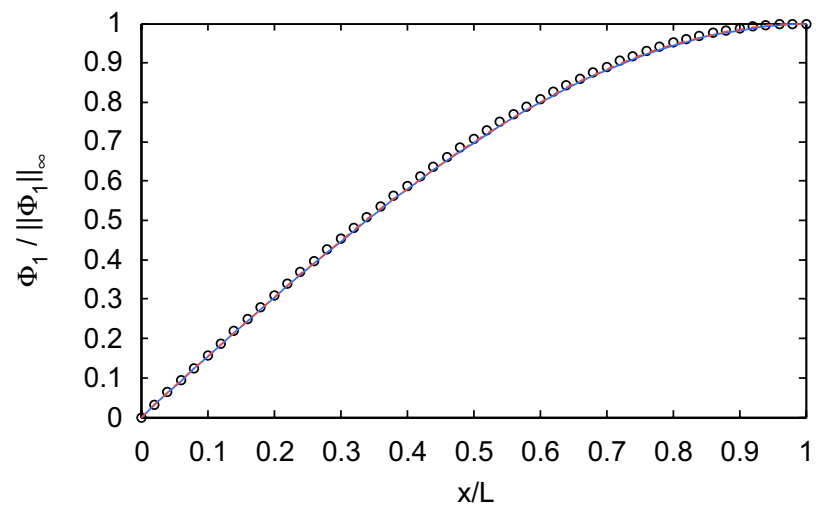

(c)

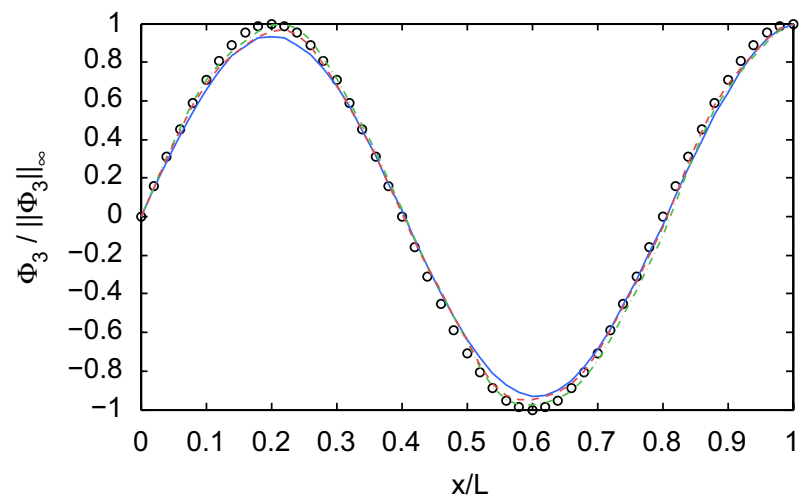

(b)

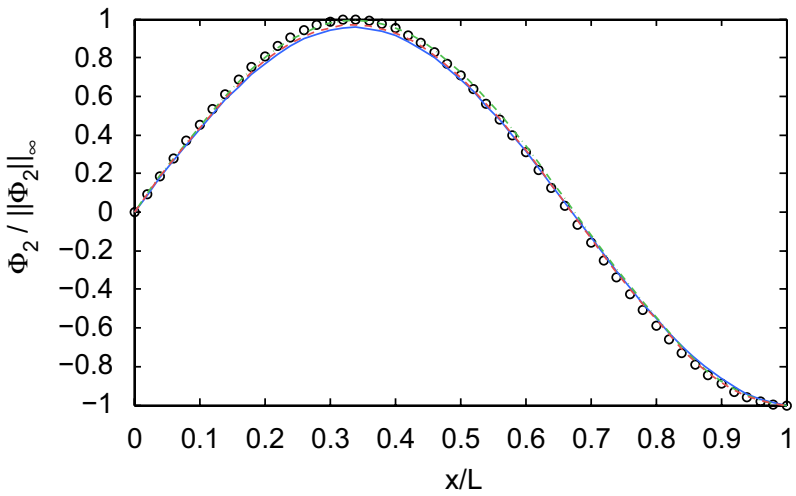

(d)

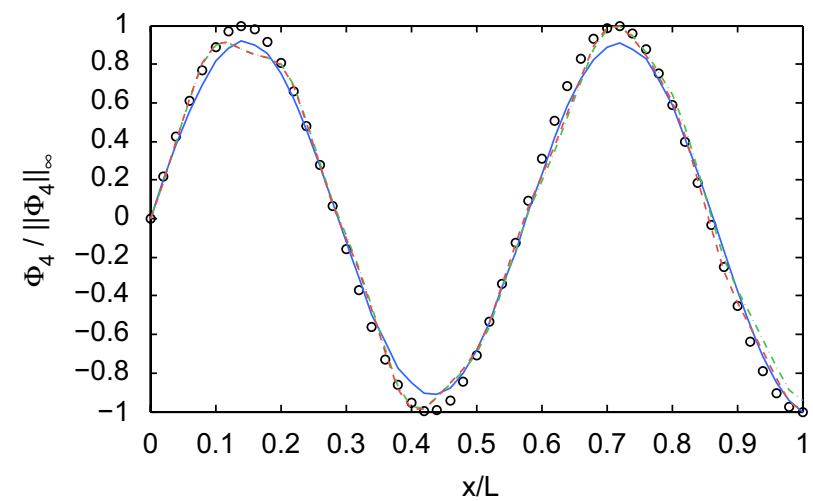

Fig. 2. Comparison of the first four POMs computed by the PODa (-), the PODd (---) and the PODh (--). The black circles (०) represent the rod eigenmodes $\left\{\psi_{n}\right\}_{n=1}^{4}$. 
Table 1

Comparison of the infinite reconstruction errors $\varepsilon_{\infty}$ computed over 5 or 10 fundamental periods of oscillation for each POD formulation

\begin{tabular}{llll}
\hline & PODa & PODh & PODd \\
\hline 5 periods & 1.89 & 2.39 & 3.74 \\
10 periods & 2.76 & 3.58 & 4.44 \\
\hline
\end{tabular}

(a)

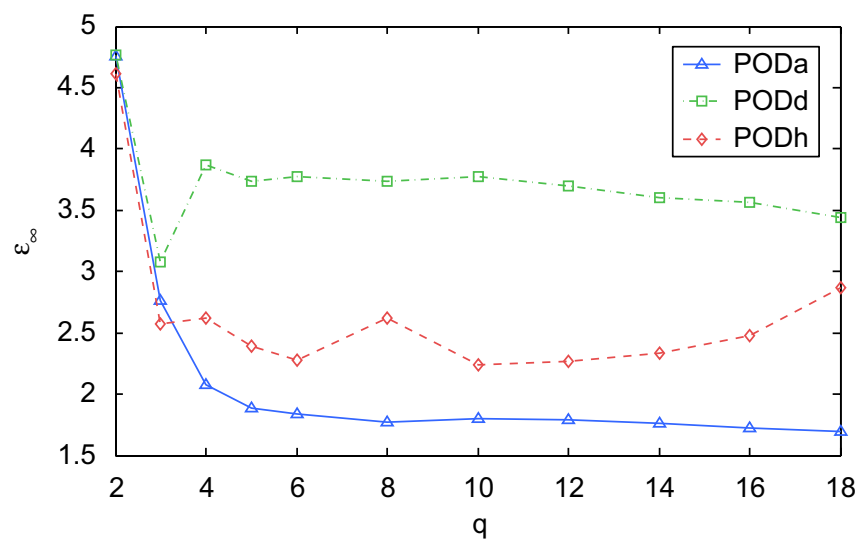

(b)

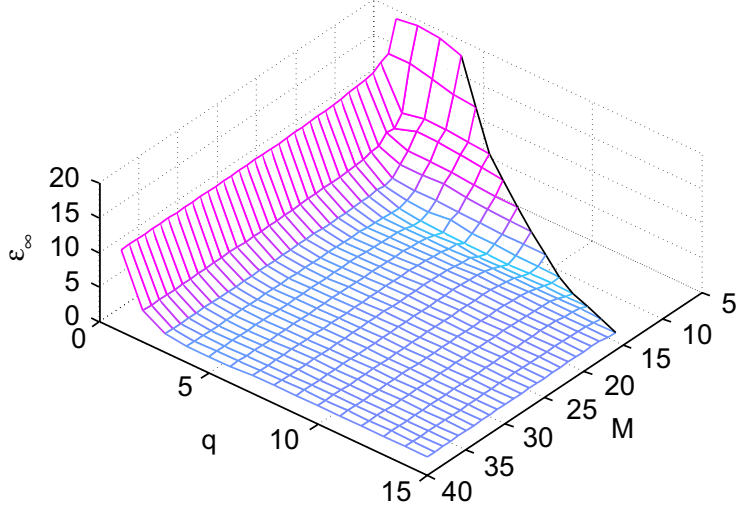

Fig. 3. Convergence of the infinite reconstruction error $\varepsilon_{\infty}$ relative to the different POD formulations for the response to the initial condition in function of the number $q$ of POMs used in the POD basis (a). The plot (b) presents the convergence of $\varepsilon_{\infty}$ for the PODh when the number $q$ of POMs and the numbers of snapshots $M$ used to compute the POMs are increased.

very close to the modes of vibration as noted by Feeny and Kappagantu [8] and Kerschen and Golinval [9]. Indeed it can be demonstrated in this particular case that the POMs converge to the eigenmodes when a sufficient number of linearly independent snapshots are used in the correlation tensor.

Table 1 summarizes the values of the infinite error $\varepsilon_{\infty}$ defined in Eq. (23) and computed for each formulation over 5 and 10 fundamental periods of oscillation. It can be seen that the error produced by the PODh is comprised between the errors produced by the two other formulations. This result is interesting since it means that the error produced by the PODh has the same order of magnitude as the one of the PODd: the two formulations could therefore be used together in a coupled problem.

Fig. 3a compares the convergence behavior of each POD formulation. The PODa has the most regular convergence behavior and quickly reaches a small asymptotic error close to $1.7 \%$. The PODd is characterized by an infinite error which is slowly decreasing when the number of POMs used in the reduced-order model is increased. Finally, the PODh has a more irregular convergence with small localized peaks of error. However, the mean error is acceptable and for a relatively small number of POMs, this formulation is a good compromise between the PODa and the PODd.

The second graph Fig. $3 \mathrm{~b}$ describes the convergence of the infinite error for the PODh when the number of POMs $q$ and the number of snapshots $M$ used to compute the POMs are increased. The graph is truncated when $q \geqslant M$ since the number of POMs extracted from the correlation tensor is limited by its rank, itself being equal to the number of snapshots $M$ when the snapshots have been chosen to be linearly independent like here. It is clear that when $q$ and $M$ increase, an asymptotic error is reached about $\varepsilon_{\infty} \approx 2.7 \%$. However, the minimal error encountered is not the asymptotic one but is found for $(M, q)=(13,9)$ where $\varepsilon_{\infty}=1.23 \%$. This means that it is not necessary to increase too much the number of snapshots or POMs in the reduced-order model to obtain an accurate response. Indeed, with only 5 POMs, $99.98 \%$ of the energy contained in the system is captured by the projection basis and additional POMs added to the projection basis are always associated with smaller eigenvalues which are contaminated with round-off errors preventing an improvement of the accuracy. 
A more theoretical reason proposed by Rempfer [19] is that while the local dynamics is guaranteed to improve by increasing the number of POMs, the global dynamical behavior of the system is not always preserved by the POD-Galerkin approach.

\section{Hybrid POD formulations for the response to a prescribed displacement}

The three preceding formulations have been presented for the free response to an initial condition for the sake of simplicity although they are not restricted to it and could be extended to the forced response with some additional work. The forced response considered here involves a displacement imposed at the free end of the rod (Dirichlet boundary condition). The displacement $\gamma(t)$ applied at the free end modifies the boundary conditions in

$$
u(x=L, t)=\gamma(t) \quad \forall t>0
$$

The Dirichlet boundary condition at the free-end is non-homogeneous and consequently the POMs no longer inherit this property: the boundary conditions which were implicitly satisfied by the reduced-order model through the POMs for the free response must now be explicitly imposed. Several techniques are presented in the following for the hybrid POD formulation but all of them could also be applied to the discrete POD.

\subsection{The penalty method (PM)}

The PM is often used to impose Dirichlet boundary conditions in structural problems [20] and has already been used by Tang et al. [15] in the context of POD reduced-order model. The principle consists in replacing the strong Dirichlet boundary condition $u(x=L, t)=\gamma(t)$ by the artificial Neumann boundary condition:

$$
\left.\frac{\partial u}{\partial x}\right|_{x=L}=\frac{u_{L}-\gamma(t)}{\varepsilon}
$$

where $\varepsilon$ is a parameter small enough to satisfy approximately the boundary condition with $u_{L}=u(x=L, t)$. We define the penalty factor $f_{p}=c^{2} / \varepsilon$ whose value has to be great so as to $u_{L}=\gamma(t)$ when $f_{p} \rightarrow \infty$. The artificial Neumann boundary condition can be explicitly introduced in the reduced-order model by performing one parts integration in Eq. (15). However, unlike the free response, the boundary terms do not all vanish: only the one corresponding to the homogeneous boundary condition at the clamped side is equal to zero. After reorganization of the terms, the reduced-order model becomes:

$$
\left.\rho S \frac{\mathrm{d}^{2} a_{j}}{\mathrm{~d} t^{2}}=-E S \sum_{k=1}^{q}\left\langle\frac{\mathrm{d} \varphi^{(k)}}{\mathrm{d} x}, \frac{\mathrm{d} \varphi^{(j)}}{\mathrm{d} x}\right\rangle a_{k}-E S\left\langle\frac{\mathrm{d} \bar{u}}{\mathrm{~d} x} \frac{\mathrm{d} \varphi^{(j)}}{\mathrm{d} x}\right\rangle+\left.E S \sum_{k=1}^{q} \frac{\mathrm{d} \varphi^{(k)}}{\mathrm{d} x}\right|_{x=L} a_{k}+\left.\frac{\mathrm{d} \bar{u}}{\mathrm{~d} x}\right|_{x=L}\right) \varphi^{(j)}(x=L)
$$

The term in brackets is in fact the spatial derivative of the variable $u$ evaluated in $x=L$ when $u$ is decomposed by the POD with Eq. (11). The artificial boundary condition Eq. (25) can then be replaced in Eq. (26):

$$
\frac{\mathrm{d}^{2} a_{j}}{\mathrm{~d} t^{2}}=-c^{2} \sum_{k=1}^{q}\left\langle\frac{\mathrm{d} \varphi^{(k)}}{\mathrm{d} x}, \frac{\mathrm{d} \varphi^{(j)}}{\mathrm{d} x}\right\rangle a_{k}-c^{2}\left\langle\frac{\mathrm{d} \bar{u}}{\mathrm{~d} x}, \frac{\mathrm{d} \varphi^{(j)}}{\mathrm{d} x}\right\rangle+f_{p}\left(u_{L}(t)-\gamma(t)\right) \varphi^{(j)}(x=L)
$$

The displacement $u_{L}$ is decomposed on the POD basis with Eq. (11): $u_{L}=\overline{u_{L}}+\sum_{k=1}^{q} a_{k}(t) \varphi^{(k)}(x=L)$. The vector $\boldsymbol{\varphi}_{L}=\left[\varphi^{(1)}(x=L) \cdots \varphi^{(q)}(x=L)\right]^{\mathrm{T}}$ is introduced so that the sum can be interpreted as the vector product $\varphi_{L}^{\mathrm{T}} \mathbf{a}$ where $\mathbf{a}$ is the vector of the modal amplitudes. The set of equations Eqs. (27) can be grouped in only one matricial equation $\ddot{\mathbf{a}}=\widetilde{\mathscr{L}} \mathbf{a}+\widetilde{\mathscr{K}}$ such that

$$
\begin{aligned}
& \widetilde{\mathscr{L}}=\mathscr{L}+\mathscr{L}_{p} \quad \text { with } \mathscr{L}_{p}=f_{p}\left(\boldsymbol{\varphi}_{L} \boldsymbol{\varphi}_{L}^{\mathrm{T}}\right) \\
& \widetilde{\mathscr{K}}=\mathscr{K}+\mathscr{K}_{p} \quad \text { with } \mathscr{K}_{p}=f_{p}\left(\overline{u_{L}}-\gamma(t)\right) \varphi_{L}
\end{aligned}
$$

where the matrix $\mathscr{L}$ and the vector $\mathscr{K}$ are exactly the same as those found in Eq. (17) for the free response with the hybrid formulation. The remaining terms can be considered as penalty terms whose action is to enforce the boundary condition. 


\subsection{The Lagrange multiplier method (LMM)}

In this case, the displacement imposed at the free end is treated as a constraint which has to be added to the equation governing the rod. The displacement generates an unknown reaction $R_{\gamma}$ that should be introduced in the second member of Eq. (12). This reaction $R_{\gamma}$ is an additional unknown reflecting the constraint of the imposed displacement and is thus the Lagrange multiplier of the following problem:

$$
\left\{\begin{array}{l}
\rho S \frac{\partial^{2} u}{\partial t^{2}}-E S \frac{\partial^{2} u}{\partial x^{2}}=R_{\gamma} \\
u(x=L, t)=\gamma(t)
\end{array}\right.
$$

The first equation is, like before, projected onto each POM after having substituted $u$ by its decomposition Eq. (11) on the POD basis. In the second equation, the variable $u$ is also replaced with the same decomposition evaluated in $x=L$ :

$$
\left\{\begin{array}{l}
\rho S \frac{\mathrm{d}^{2} a_{j}}{\mathrm{~d} t^{2}}=E S \sum_{k=1}^{q}\left\langle\frac{\mathrm{d}^{2} \varphi^{(k)}}{\mathrm{d} x^{2}}, \varphi^{(j)}\right\rangle a_{k}+E S\left\langle\frac{\mathrm{d}^{2} \bar{u}}{\mathrm{~d} x^{2}}, \varphi^{(j)}\right\rangle+\left\langle R_{\gamma}, \varphi^{(j)}\right\rangle \\
u(x=L, t)=\overline{u_{L}}+\sum_{k=1}^{q} a_{k}(t) \varphi^{(k)}(x=L)=\gamma(t)
\end{array}\right.
$$

Since the reaction $R_{\gamma}$ acts in fact only at the free end, it can be defined by a Dirac distribution: $R_{\gamma}=r_{L} \delta(x-L)$, where the scalar $r_{L}$ represents the intensity of the reaction at $x=L$. Consequently, the inner product involving the reaction $R_{\gamma}$ is simplified in: $\left\langle R_{\gamma}, \varphi^{(j)}\right\rangle=\int_{0}^{L} r_{L} \delta(x-L) \varphi^{(j)}(x) \mathrm{d} x=r_{L} \varphi^{(j)}(x=L)$. The vector of the reaction projected on each POM is given by $r_{L} \varphi_{L}$, where $\varphi_{L}$ is the vector defined in the previous paragraph and containing the values of the POMs at the free end. This vector is also used to rewrite the second line in Eq. (30) in $\overline{u_{L}}+\varphi_{L}^{\mathrm{T}} \mathbf{a}=\gamma(t)$. The reduced-order model becomes:

$$
\left\{\begin{array}{l}
\ddot{\mathbf{a}}=\breve{\mathscr{L}} \mathbf{a}+\check{\mathscr{K}}+r_{L} \boldsymbol{\varphi}_{L} \\
0=\boldsymbol{\varphi}_{L}^{\mathrm{T}} \mathbf{a}+\bar{u}_{L}-\gamma(t)
\end{array}\right.
$$

where the general term of the matrix $\breve{\mathscr{L}}$ and the vector $\breve{\mathscr{K}}$ are given by

$$
\check{\mathscr{L}}_{i j}=c^{2}\left\langle\frac{\mathrm{d}^{2} \varphi^{(j)}}{\mathrm{d} x^{2}}, \varphi^{(i)}\right\rangle, \quad \check{\mathscr{K}}_{i}=c^{2}\left\langle\frac{\mathrm{d}^{2} \bar{u}}{\mathrm{~d} x^{2}}, \varphi^{(i)}\right\rangle
$$

Finally the reduced-order model is formulated for the augmented variable vector $\widetilde{\mathbf{a}}=\left[\mathbf{a} r_{L}\right]^{\mathrm{T}}$ and takes the new form $\widetilde{\mathscr{I}} \ddot{\mathbf{a}}=\widetilde{\mathscr{L}} \widetilde{\mathbf{a}}+\widetilde{\mathscr{K}}$. The detailed expression of the reduced-order model is the following, where $\mathbf{I}$ is the $q \times q$ identity matrix:

$$
\left[\begin{array}{ll}
\mathbf{I} & \mathbf{0} \\
\mathbf{0} & 0
\end{array}\right]\left[\begin{array}{c}
\ddot{\mathbf{a}} \\
\ddot{r}_{L}
\end{array}\right]=\left[\begin{array}{cc}
\breve{\mathscr{L}} & \boldsymbol{\varphi}_{L} \\
\boldsymbol{\varphi}_{L}^{\mathrm{T}} & 0
\end{array}\right]\left[\begin{array}{c}
\mathbf{a} \\
r_{L}
\end{array}\right]+\left[\begin{array}{c}
\breve{\mathscr{K}} \\
\overline{u_{L}}-\gamma(t)
\end{array}\right]
$$

\subsection{The control function method (CFM)}

The last technique presented in this paper to take into account the non-homogeneous boundary condition is the CFM commonly used to build reduced-order models for active control in flow dynamics, see Ref. [15] for example. The variable $u$ is divided into two parts, the first verifying homogeneous boundary conditions and denoted $u_{h}$ and the second representing the effects of the forcing term and denoted $u_{f}$, such that $u=u_{h}+u_{f}$ with $u_{h}(x=L, t)=0$ and $u_{f}(x=L, t)=\gamma(t)$.

The POMs are computed for the centered homogeneous snapshots $s_{h}^{(k)}=u_{h}^{(k)}-\overline{u_{h}}$ and the POD decomposition is thus $u_{h}(x, t)=\overline{u_{h}}(x)+\sum_{k=1}^{q} a_{k}(t) \varphi_{h}^{(k)}(x)$. The notation $\varphi_{h}^{(k)}$ is now used to highlight the homogeneous character of the POMs derived from the snapshots that have been homogenized. The previous decomposition of $u_{h}$ can be replaced in the relation $u=u_{h}+u_{f}$ to obtain the POD decomposition of the 
inhomogeneous variable $u$. Before doing this, it is interesting to write the forcing term $u_{f}$ as the product of a spatial and a temporal function which corresponds to the temporal modulation of the boundary condition Eq. (24): $u_{f}(x, t)=\gamma(t) u_{c}(x)$. The new POD decomposition for the variable $u$ is then:

$$
u(x, t)=\overline{u_{h}}(x)+\gamma(t) u_{c}(x)+\sum_{k=1}^{q} a_{k}(t) \varphi_{h}^{(k)}(x)
$$

The forcing term is not completely defined as the shape of the spatial control function $u_{c}$ has not yet been given. Several choices are possible since the only constraint imposed by $u_{f}(x=L, t)=\gamma(t)$ is that $u_{c}(x=L)=1$. A Dirac distribution $u_{c}(x)=\delta(x-L)$ satisfies the previous condition but is certainly not optimal because of the discontinuity inherent to this function. A better choice consists in choosing the static solution of the problem for a unitary displacement imposed at the free end: $u_{c}(x)=x / L$.

The reduced-order model is easily obtained by introducing the new decomposition Eq. (33) into the continuous equation of the rod and by projecting it onto each POM:

$$
\frac{\mathrm{d}^{2} a_{j}}{\mathrm{~d} t^{2}}=c^{2} \sum_{k=1}^{q}\left\langle\frac{\mathrm{d}^{2} \varphi^{(k)}}{\mathrm{d} x^{2}}, \varphi_{h}^{(j)}\right\rangle a_{k}+c^{2}\left\langle\frac{\mathrm{d}^{2} \overline{u_{h}}}{\mathrm{~d} x^{2}}, \varphi_{h}^{(j)}\right\rangle+c^{2} \gamma(t)\left\langle\frac{\mathrm{d}^{2} u_{c}}{\mathrm{~d} x^{2}}, \varphi_{h}^{(j)}\right\rangle-\frac{\mathrm{d}^{2} \gamma(t)}{d t^{2}}\left\langle u_{c}, \varphi_{h}^{(j)}\right\rangle
$$

The form $\ddot{\mathbf{a}}=\widetilde{\mathscr{L}} \mathbf{a}+\widetilde{\mathscr{K}}$ of the reduced-order model is preserved but the matrix $\widetilde{\mathscr{L}}$ and the vector $\widetilde{K}$ are now defined by

$$
\begin{aligned}
& \widetilde{\mathscr{L}}_{i j}=c^{2} \sum_{j=1}^{q}\left\langle\frac{\mathrm{d}^{2} \varphi^{(j)}}{\mathrm{d} x^{2}}, \varphi_{h}^{(i)}\right\rangle \\
& \widetilde{\mathscr{K}}_{i}=c^{2}\left\langle\frac{\mathrm{d}^{2} \overline{u_{h}}}{\mathrm{~d} x^{2}}, \varphi_{h}^{(i)}\right\rangle+c^{2} \gamma(t)\left\langle\frac{\mathrm{d}^{2} u_{c}}{\mathrm{~d} x^{2}}, \varphi_{h}^{(i)}\right\rangle-\frac{\mathrm{d}^{2} \gamma(t)}{\mathrm{d} t^{2}}\left\langle u_{c}, \varphi_{h}^{(i)}\right\rangle
\end{aligned}
$$

The three formulations given by Eqs. (28), (32) and (35) are clearly different. The PM is very easy to implement because the matrix and vector of the reduced-order model are the same as those obtained for the free response; they are only modified by the addition of a penalty matrix (or vector) which is easily computed once the POMs are known. The main drawback of the method is that the boundary condition is only approximated and not exactly satisfied. Indeed, the greater the penalty factor, the more exact the boundary condition but the more ill-conditioned the matrices. The Lagrange multiplier method (LMM) leads to the introduction of a new variable in the system which changes the form of the reduced-order model: indeed, the matrix $\widetilde{I}$ in the left member is singular and prevents finding again the same form common to the other formulations. Finally, the CFM requires more modifications since the snapshots have to be first homogenized before computing the POMs. Another assumption is that the forcing term can be decomposed as the product of a spatial and a temporal function. The reduced-order model derived with the new decomposition keeps the same usual form but involves time derivatives of the boundary condition time modulation $\gamma(t)$. The acceleration associated to the imposed displacement has to be known and this could sometimes be a drawback of the method.

\subsection{Hybrid POD evaluation for the response to a prescribed displacement}

The time integration of the reduced-order model is now performed either by the ODEPACK solver or with a Newmark algorithm (see Ref. [20]). This algorithm is especially adapted for the LMM where the differential equation cannot be written under the generic form $\dot{\mathbf{a}}=f(\mathbf{a}, t)$ which is solved by the ODEPACK. The efficiency of each formulation is evaluated by means of the error $\varepsilon_{\infty}$ defined in Eq. (23). The reference solutions are now the solutions computed with a finite elements simulation.

The hybrid POD reduced-order model is used to reconstruct the response of the rod with a displacement imposed at the free end. The POMs are thus evaluated from a discrete set of snapshots which have been computed by a finite elements simulation for the forced response. The POMs for the PM and LMM are identical. They are represented in Fig. 4 with the black filled circles: their shape is slightly different from the one obtained with snapshots of the free response and from the eigenmodes (see Fig. 2 for the comparison). 
(a)

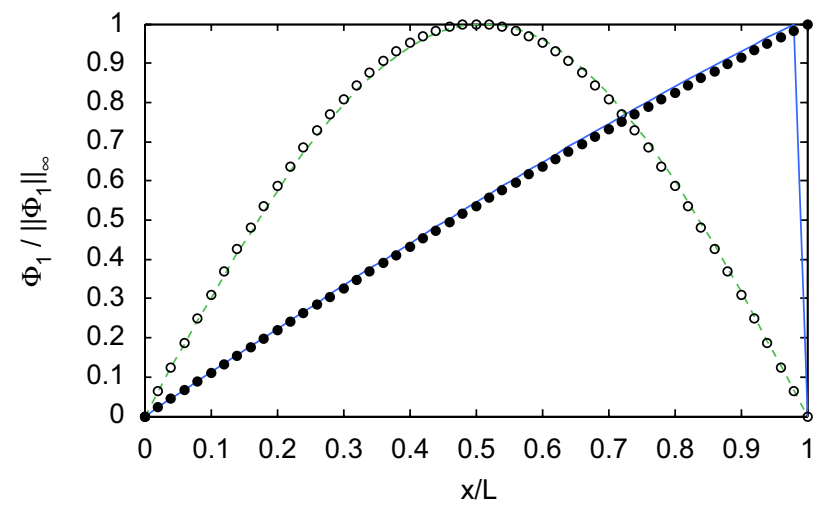

(c)

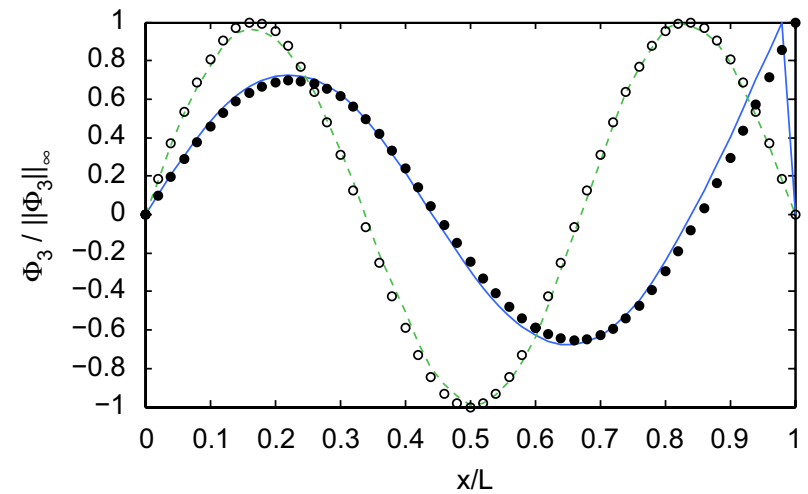

(b)

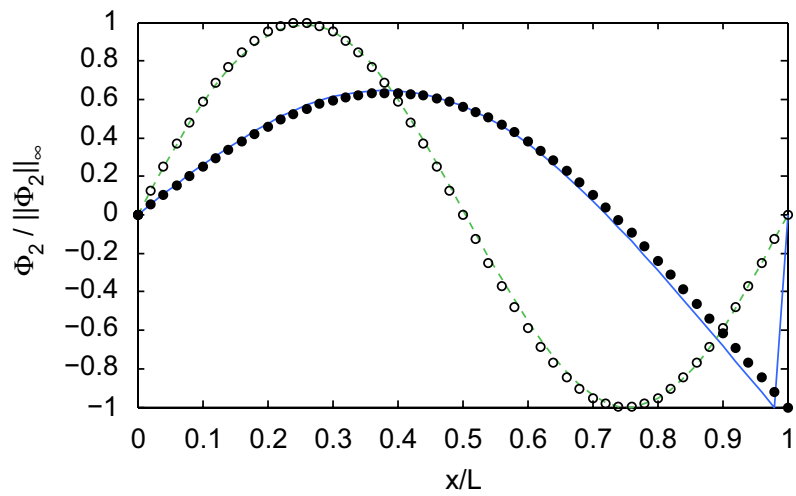

(d)

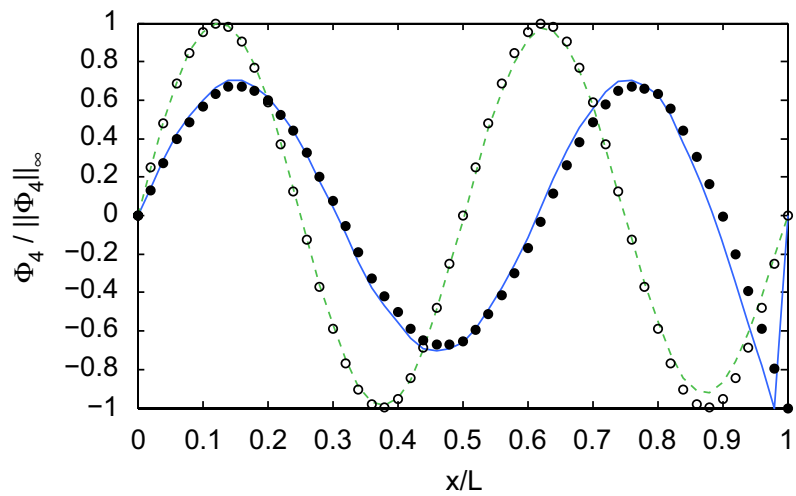

Fig. 4. Comparison of the first four POMs for the forced response. The empty black circles (o) represent the eigenmodes of the rod clamped at both ends whereas the black filled circles (•) indicates the POMs of the forced response when the PM or LMM are used to impose the displacement. The curves (-) and resp. (--) represent the shape of the POMs when the CFM is adopted and the control function is $u_{c}(x)=\delta(x-L)$ or resp., $u_{c}(x)=x / L$.

Indeed, the displacement imposed at the free end produces a reaction at this point so that the Neumann boundary condition at the free end which was homogeneous for the free response becomes now nonhomogeneous. Thus the POMs no longer satisfy an homogeneous Neumann boundary condition at the free end and the shape of the POMs therefore differs significantly in the neighborhood of the displacement application point. These POMs are used only for the PM or the LMM. Indeed, if the CFM is adopted, the snapshots have to be homogenized before computing the POMs. The shape of the POMs then depends on the control function used for the homogenization: if a Dirac function $u_{c}(x)=\delta(x-L)$ is used, the modes are similar to those of the PM or LMM except for the last point $x=L$ where the value is zero. On the contrary, with the static solution $u_{c}(x)=x / L$, the modes differ significantly from the previous ones: in this case, homogeneous boundary conditions are satisfied by the POMs at both ends of the rod but the shape does not exhibit any discontinuity. The POMs then coincide with the eigenmodes of a rod which would be clamped at both ends; these eigenmodes are represented with empty black circles in Fig. 4.

We then compare the efficiency of the three techniques (PM, LMM and CFM) to take into account a displacement $\gamma(t)=U_{e} \sin \left(2 \pi f_{e} t\right)$ imposed at the free end. Fig. 5 presents the response of the reduced-order model based on the CFM with $u_{c}(x)=x / L$ for three couples of parameters $\left(f_{e}, U_{e}\right)$. The responses computed with the other methods (PM, LMM and CFM with $\left.u_{c}(x)=\delta(x-L)\right)$ are not shown since they are not distinguishable. For each response, the POMs have been computed from snapshots stemming from the resolution of the problem with the excitation parameters $\left(f_{e}, U_{e}\right)=(150,0.01)$. This POD basis is then used to 
(a)

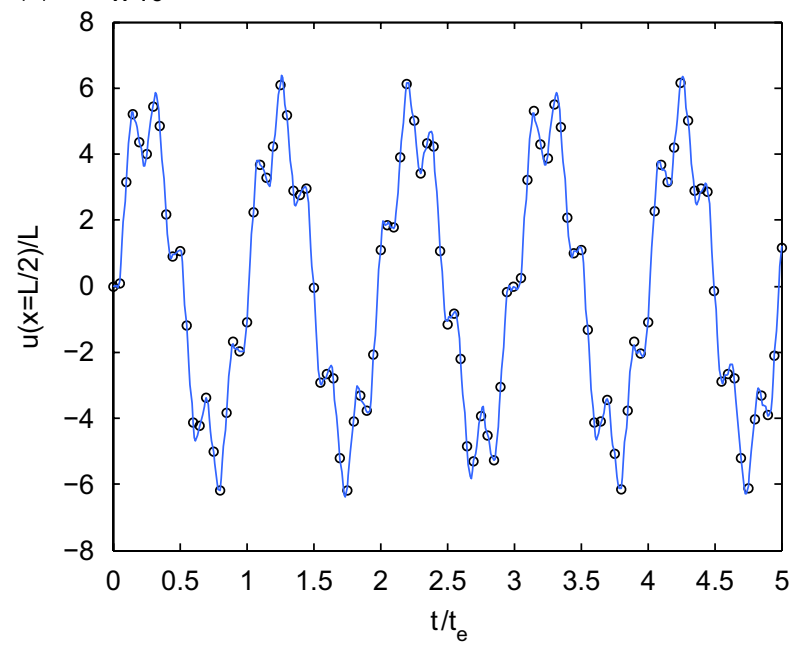

(b)

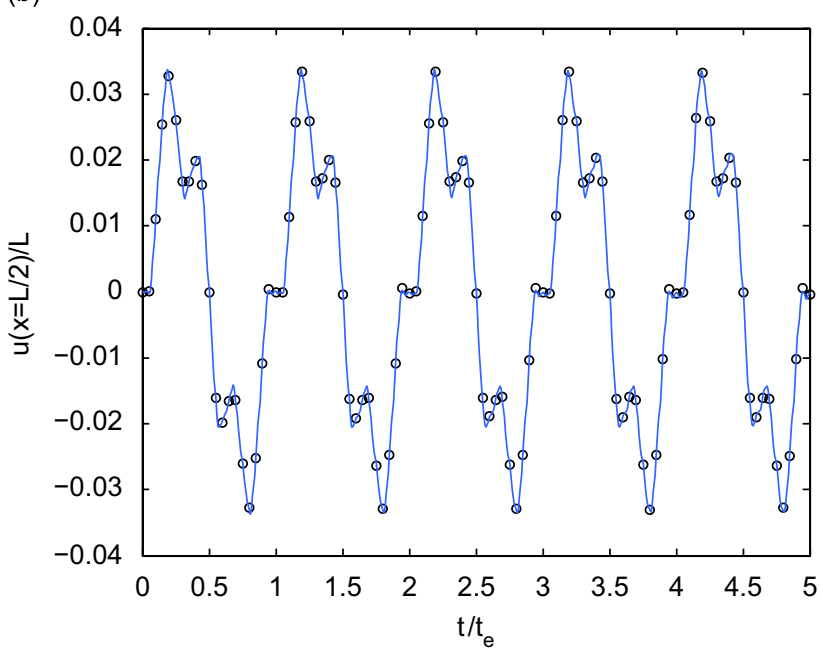

(c)

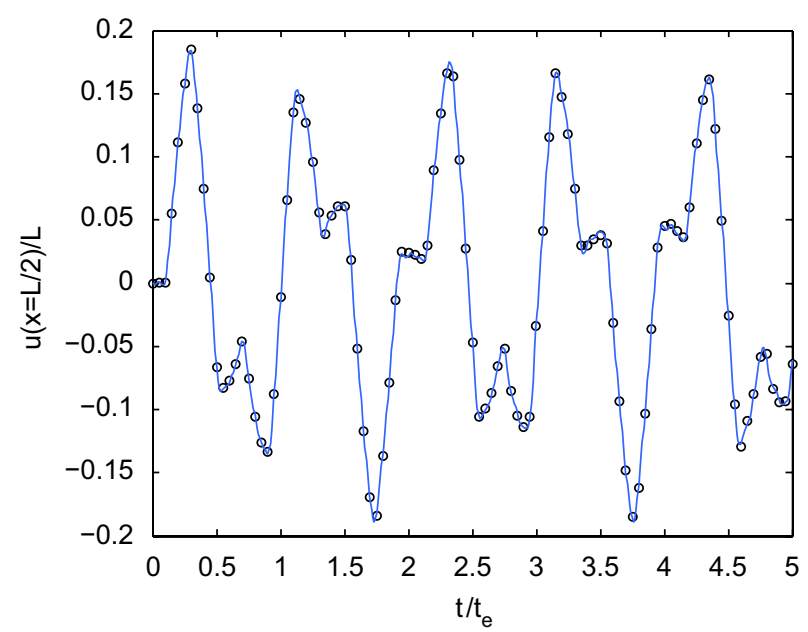

Fig. 5. Comparison between the POD responses for the forced response computed for three different couples of excitation parameters ( - ) and the reference solution (o): (a) $\left(f_{e}, U_{e}\right)=(150,0.01)$, (b) $\left(f_{e}, U_{e}\right)=(200,0.05)$, (c) $\left(f_{e}, U_{e}\right)=(325,0.2)$.

calculate the response for the three couples of parameters $\left(f_{e}, U_{e}\right)=(150,0.01),\left(f_{e}, U_{e}\right)=(200,0.05)$ and $\left(f_{e}, U_{e}\right)=(325,0.2)$ by introducing the desired imposed displacement $\gamma(t)$ characterized by each couple of parameters $\left(f_{e}, U_{e}\right)$ in Eq. (35). In each case, the response coincide exactly with the reference solution which has been computed with a finite elements simulation.

To highlight the differences between the PM, LMM and CFM, the values of the infinite error $\varepsilon_{\infty}$ are plotted in Fig. 6a. Except for the PM, the error grows as the parameters go away from those used to evaluate the POMs. This is the main limitation of the POD method: since the POD basis is constructed from snapshots, the basis vectors depend on the excitation and the reduced-order model becomes less efficient as the parameters for which the response has to be computed take away from those characterizing the excitation with which the snapshots, and consequently the POMs, have been obtained. Nevertheless, the errors remain small for this simple system and the responses obtained with the different formulations are in very good agreement with the reference solutions. The best method for this example seems to be the CFM when the POMs have been homogenized with the static solution of the system $u_{c}(x)=x / L$. However, the influence of the control function is crucial and it can be seen that the choice of the Dirac function for $u_{c}$ leads to the greatest error. 
(a)

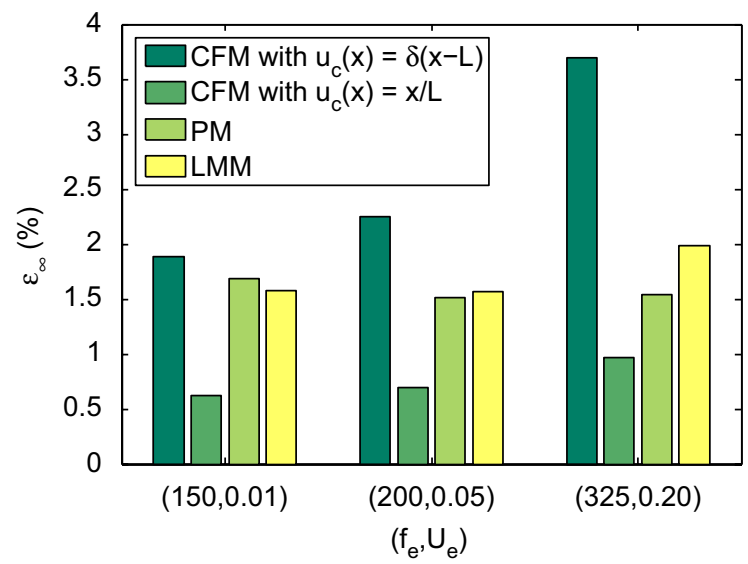

(b)

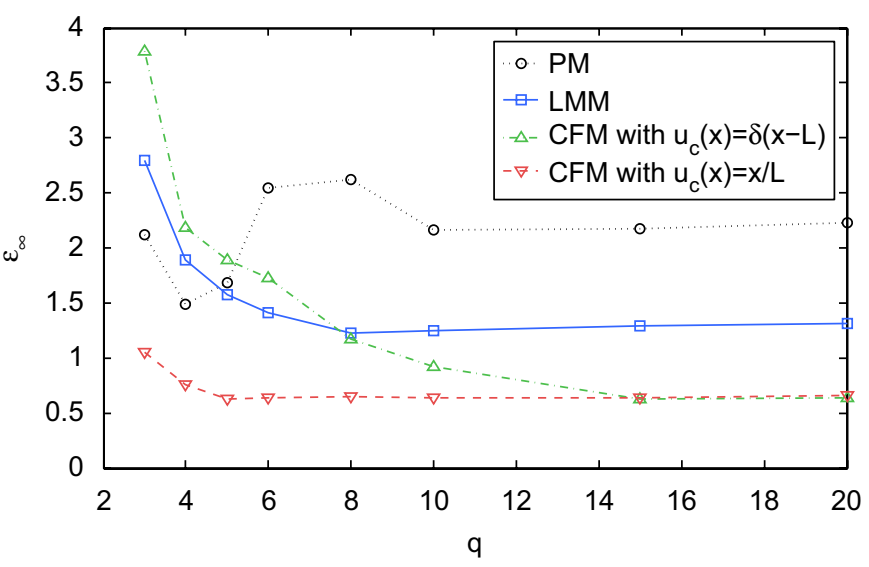

Fig. 6. Comparison of the infinite error $\varepsilon_{\infty}$ produced by the different methods used to impose the displacement: (a) for different couples of excitation parameters $\left(f_{e}, U_{e}\right)$, and (b) when the number of POMs used in the reduced-order model is increased.

Fig. $6 \mathrm{~b}$ represents for each method the convergence of the infinite reconstruction error $\varepsilon_{\infty}$ when the number $q$ of POMs contained in the POD basis is increased. The PM has in fact the greatest asymptotic error and does not converge very well. The best method is actually the CFM with the static solution, but when the number of POMs is increased, we notice that the use of the Dirac function becomes a reliable choice, better than the PM or even the LMM. This is an interesting result since the use of a Dirac does not require the computation of a physical solution of the system like it is the case with the static solution, whose determination could become time-consuming when the number of dof at the boundary is great.

The CFM seems to be the most efficient method but the choice of the control function used for the homogenization is capital. Moreover, when a displacement $u_{L}$ is imposed at the free end, it is necessary to know the acceleration $\ddot{u}_{L}$ associated to this displacement in order to evaluate the coefficients $\widetilde{\mathscr{K}}_{i}$ of the reduced-order model defined in Eq. (35). It is trivial to determine the acceleration for a sinusoidal displacement like the one imposed here, but some difficulties can arise if other shapes are used (e.g. a sawtooth or crenel function which is not derivable). Sometimes, the displacement is also not explicitly known (e.g. the case of a coupled fluid-structure system) and determining the acceleration is not obvious. The PM and LMM avoid this drawback and have besides the advantage to be more flexible: the POD basis used in the reduced-order model can eventually come from snapshots corresponding to the free response, or the forced response with a force (instead of a displacement) imposed at the free end. This could also be done with the CFM but it requires the determination of the adequate time modulations $\gamma(t)$ in order to homogenize the snapshots.

\section{Robustness of the hybrid POD method}

In this section, the robustness of the hybrid POD formulation is investigated: the reduced-order model is used to rebuild diverse responses with POMs stemming from snapshots computed for a different type of excitation. The objective is to control that the POD basis is, like the traditional eigenmode basis, suitable to rebuild responses which are very different from the one used to obtain the POMs. This is not obvious for the POD since the POMs are computed from snapshots which could have been produced by any type of excitation, whereas the eigenmodes are always obtained by solving an eigenvalue problem formed with the conservative part of the elastic system for small harmonic motion.

The first example consists in computing the response of the rod to an initial displacement $U_{0}$ at the free end when structural damping has been introduced in the system. As the continuous equation of the system is required to work with the PODh formulation, we choose a constant damping coefficient $\xi$ acting uniformly at each point of the rod to simplify the equation. The continuous equation (12) is then modified by the addition 
of a non-conservative term proportional to the velocity:

$$
\rho S \frac{\partial^{2} u}{\partial t^{2}}+\xi \frac{\partial u}{\partial t}-E S \frac{\partial^{2} u}{\partial x^{2}}=0
$$

The POMs computed from damped snapshots for the initial condition $U_{0}$ are nearly identical to the POMs of the free response without damping and consequently to the eigenmodes of the rod. This is in agreement with the results of Kerschen and Golinval [9] who found that the POMs still converge to the eigenmodes when the damping is weak and in absence of any external solicitation. Therefore, the POMs obtained with conservative snapshots can be used to rebuild the non-conservative response and vice versa since the shape of the POMs are very close in both cases. Fig. 7 presents the response obtained by the PODh for the damped system with 5 POMs computed from snapshots of the conservative response. The agreement between the rebuilt and reference solutions is excellent and the error remains very small: the value of the infinite error for the damped response is $\varepsilon_{\infty}=2.35 \%$ when non-conservative snapshots are used and falls to $\varepsilon_{\infty}=1.90 \%$ with conservative snapshots. Similarly, the undamped response to the same initial condition (identical to the response studied in Section 3.4) can be rebuilt from different sets of snapshots: the error produced is $\varepsilon_{\infty}=2.81 \%$ when nonconservative snapshots are used and falls to $\varepsilon_{\infty}=2.39 \%$ with conservative snapshots.

The second example concerns a clamped-free rod excited by a force $F$ whose application point is distant of $x_{f}<L$ from the clamped end. An obstacle is placed at the distance $e$ from the free end (see Fig. 8). When the magnitude of the force is enough important, the free end section hits the obstacle; this generates vibro-impacts and therefore produces a reaction at this point. The reaction is nonlinear and defined by $R\left(u_{L}\right)=$ $-K_{\mathrm{obs}}\left(u_{L}-e\right)$ if $u_{L}>e$ and $R\left(u_{L}\right)=0$ if $u_{L} \leqslant e$. The excitation force is sinusoidal and characterized by $F(t)=F_{e} \sin \left(2 \pi f_{e} t\right)$. According to the values of $F_{e}$ and $f_{e}$, the free end hits or not the obstacle, whose stiffness has a great value $\left(K_{\mathrm{obs}}=10^{20}\right)$ in order to model a rigid obstacle.

This second example is more interesting since the POMs are significantly different from those of the free or forced response. Fig. 9 gives the shape of the POMs obtained from a set of snapshots computed with or without the obstacle. In both cases, the excitation force $F$ is characterized by $\left(f_{e}, F_{e}\right)=(175,2.0 \mathrm{E} 6)$. When the rod hits the obstacle, the nonlinear reaction at the free end leads to a more complex response of the system

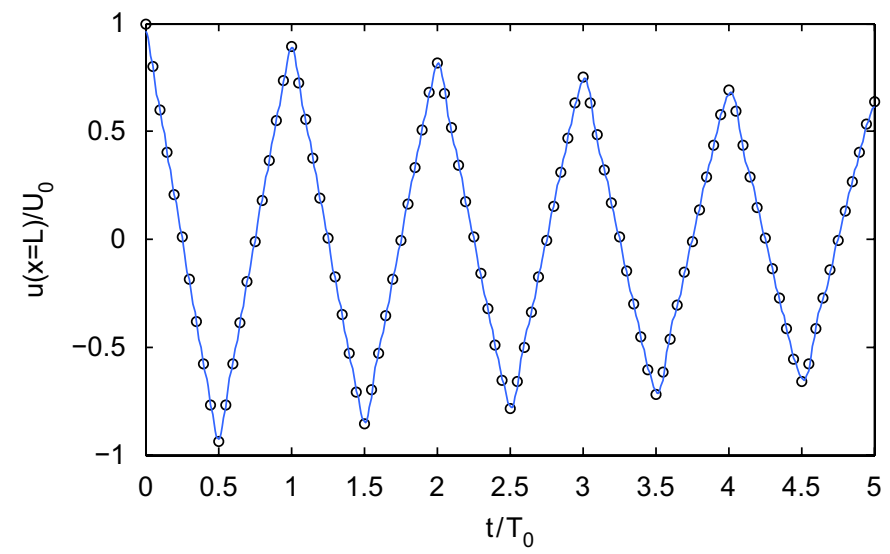

Fig. 7. Comparison between the PODh response $(-)$ and the reference solution $(\circ)$ for the rod with structural damping.

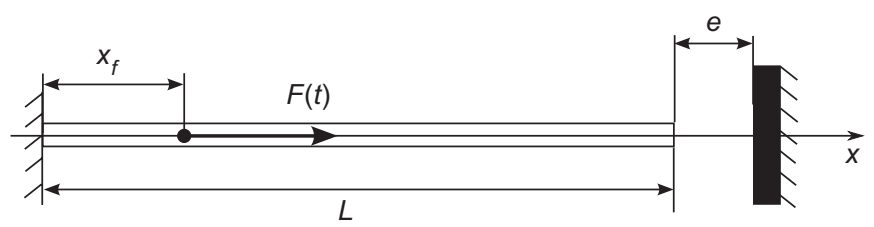

Fig. 8. Representation of the clamped-free rod excited by the force $F$ and subject to vibro-impacts due to the obstacle. 
(a)

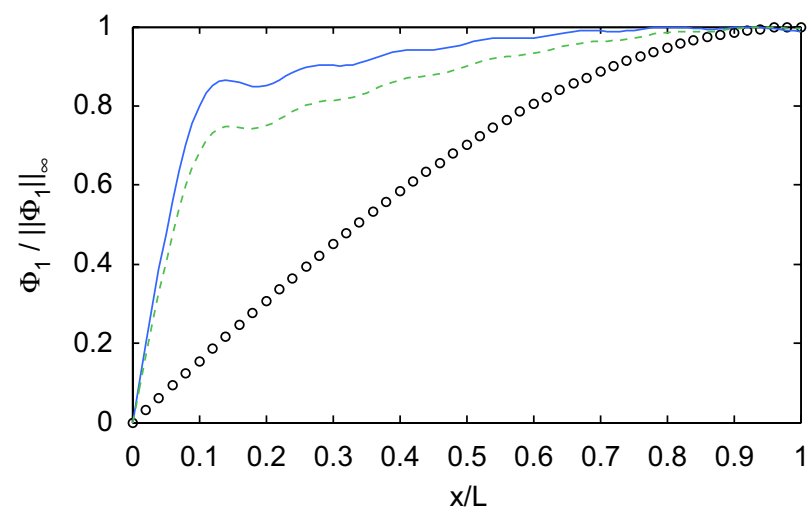

(c)

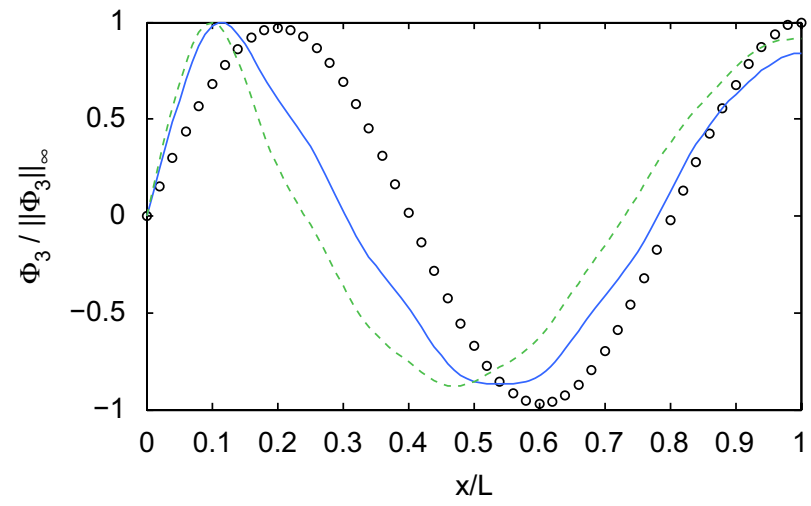

(b)

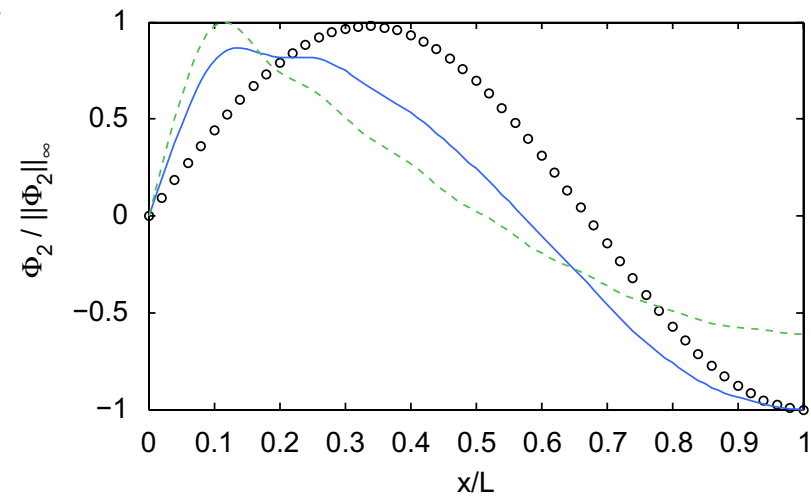

(d)

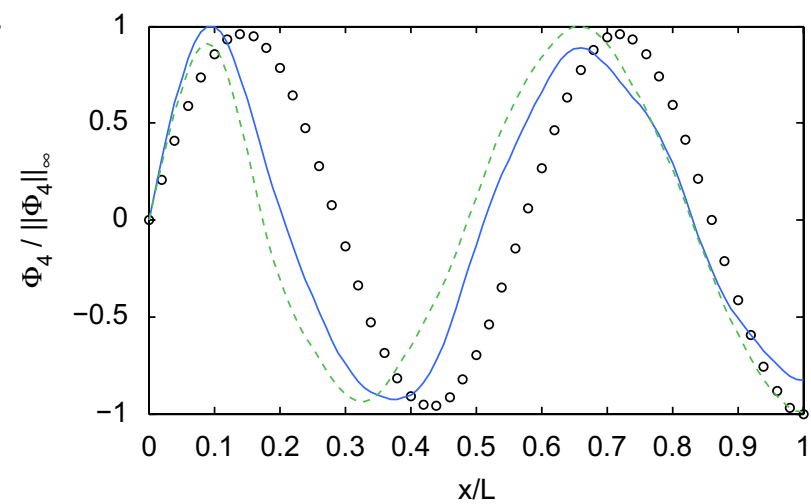

Fig. 9. Panels (a)-(d) show the first four POMs of the rod subject to vibro-impacts and computed from snapshots with the obstacle (-) or without it (--). The black circles (o) represent the rod eigenmodes.

than when there is no obstacle. In both cases (with or without the obstacle), the shape of the POMs is very different from the rod eigenmodes: the force $F$ applied at $x_{f}=0.1 L$ deforms considerably the shape of the modes in the neighborhood of this point.

The response of the system for different couples of parameters $\left(f_{e}, F_{e}\right)$ is then computed. Fig. 10 shows the responses when the POMs used in the reduced-order model have been evaluated from snapshots with the obstacle. For the first (a) and last (c) cases, the magnitude of the imposed force $F_{e}$ is great enough so that the free end hits the obstacle, whereas in the intermediate case (b) there is no contact between the free end and the obstacle. Although the POMs stem from snapshots with vibro-impacts, they are suitable to rebuild the responses either with or without contact. Indeed, the global shape of the response is in each case faithful to the reference solution, except during the phase of contact where the number and amplitude of the vibro-impacts are not exactly reproduced. However, it should be mentioned that the time step used for the time integration of the reduced-order model is ten times greater than the one used to compute the reference solution and this could explain why all of the impacts are not captured.

Although Fig. 10 exhibits a somewhat good accuracy of the response, the errors produced become now significant, notably when vibro-impacts occur. The errors are plotted in Fig. 11 for the three couples of parameters and according to the nature of the POMs used in the reduced-order model. When the free end hits the obstacle (first and last cases), the error is almost the same, whatever the nature of the POMs: despite slight differences appear according to the type of the POMs used (with or without vibro-impacts contained in the snapshots), the error has the same order of magnitude. On the contrary, if no contact happens, the POMs computed from snapshots without the obstacle are clearly more efficient than the others. The POMs of the free 
(a)

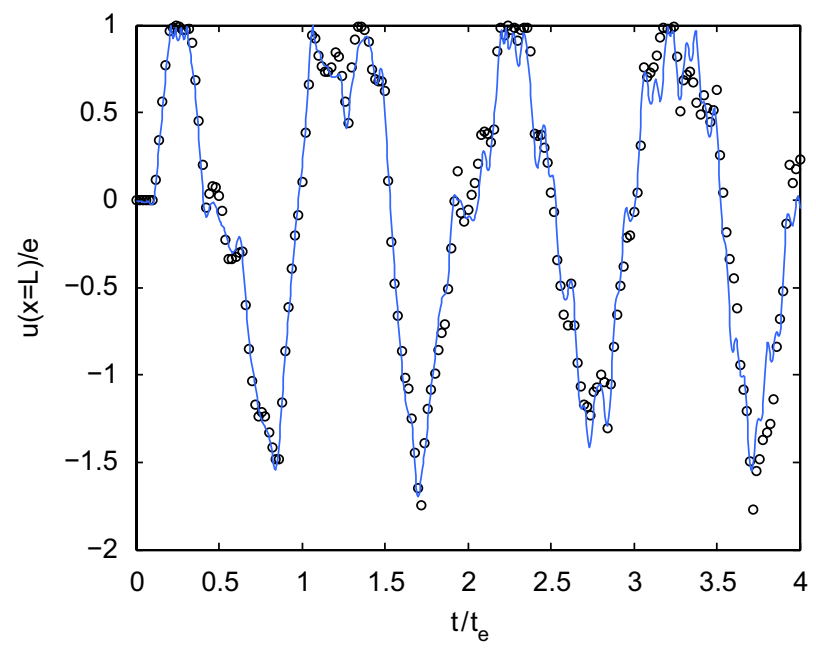

(b)

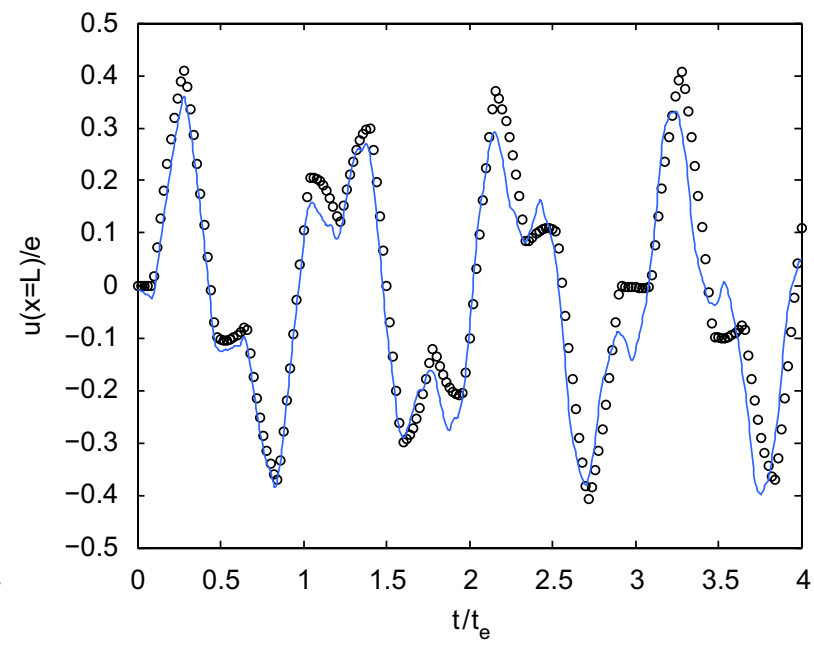

(c)

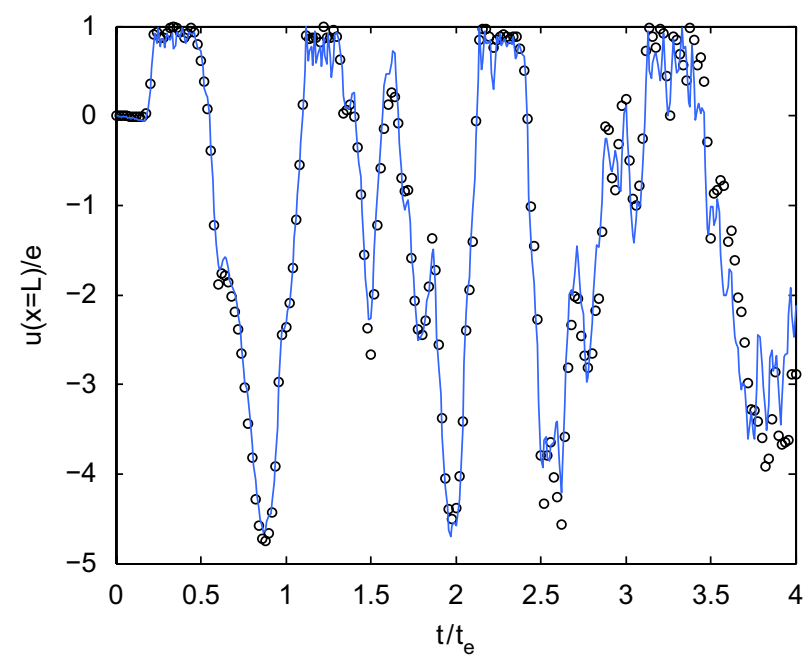

Fig. 10. Comparison between the responses for the rod subject to vibro-impacts computed for three different couples of excitation parameters (-) and the reference solution (o): (a) $\left(f_{e}, F_{e}\right)=(175,2.0 \mathrm{E} 6)$, (b) $\left(f_{e}, F_{e}\right)=(150,0.5 \mathrm{E} 6)$, (c) $\left(f_{e}, F_{e}\right)=(300,5.0 \mathrm{E} 6)$.

motion give the greatest error when no contact happens, however the results are in good agreement in other cases.

To conclude, the POD method provides a rather robust mean to build a reduced-order model: when the response is computed with POMs which have been evaluated for the same type of excitation, the accuracy is in general very good. However, the use of POMs stemming from a different type of excitation is a more delicate issue. The POMs of the free response could be employed easily to rebuild responses for other types of excitations (e.g. response with structural damping or with a nonlinear reaction induced by an obstacle, response to an imposed displacement with the PM or LMM) but this is somewhat obvious since in this particular case the POMs are close to the eigenmodes of the system. However, if we want to rebuild the free response to an initial condition, the use of POMs obtained from snapshots with a displacement or a force imposed is not appropriate since the POMs do not satisfy the suitable boundary conditions and the reducedorder model has to be adapted: the simplification of the boundary terms appearing in the parts integration of is no longer possible. 


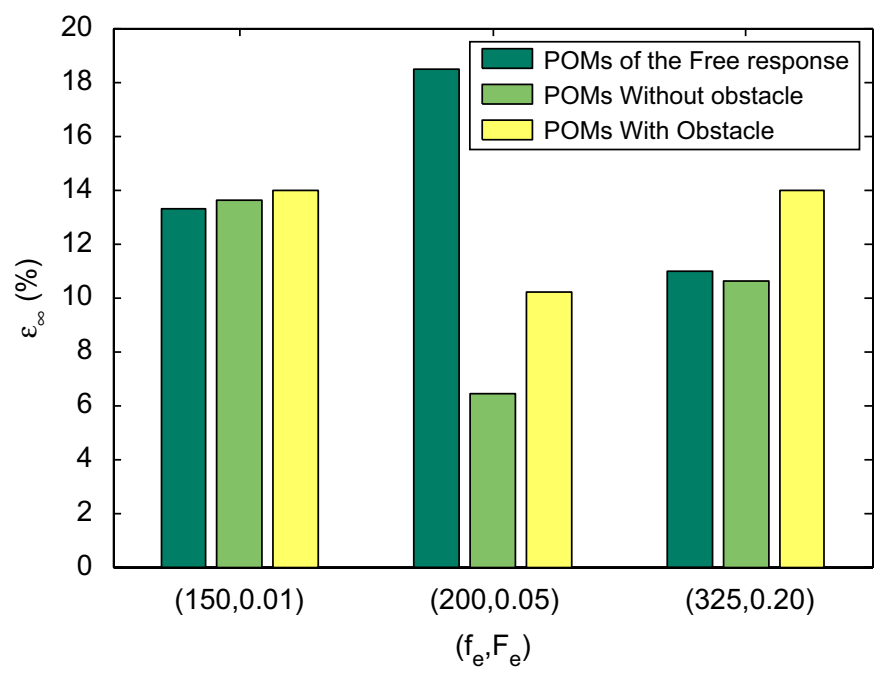

Fig. 11. Comparison of the infinite reconstruction error $\varepsilon_{\infty}$ for different couples of parameters according to the nature of the POMs used.

\section{Conclusion}

In this paper the hybrid POD formulation has been evaluated and compared for the response to an initial condition to two other POD formulations, namely the discrete and the analytical POD formulations. The hybrid POD is suitable to build reduced-order models leading to a small reconstruction error, and only small differences have been highlighted between the three formulations. The comparison revealed that the analytical formulation was the most accurate but its use is limited to the present system and only serves as reference. The hybrid formulation represents an interesting alternative to the discrete approach and is particularly useful when the discrete equation is not available. An important conclusion is that the hybrid approach (PODh) which is usually adopted for fluid mechanics generates a response whose error is of the same order as the one produced by the discrete approach (PODd) which is commonly used for structural mechanics. This means that the PODh and the PODd could be employed together to build a reduced-order model for a coupled fluid-structure system where the fluid would be reduced by the PODh and the structure by the PODd.

The second important point concerns the treatment of prescribed displacements by the hybrid POD reduced-order model. Several methods have been compared and advantages and drawbacks have been highlighted. Except the PM which has a surprising convergence behavior when the number of POMs in the projection basis is increased, the other methods (LMM and CFM) behave well and the asymptotic error remains small. The CFM is the most accurate but has several drawbacks, particularly the sensitivity to the control function or the need for the time derivative of the excitation function. However, once a good control function has been determined, the method is robust and converge very quickly as the number of POMs is increased. The LMM has also proved to be efficient, but the asymptotic error of reconstruction is slightly greater.

To conclude, the investigations conducted here show that the hybrid POD approach is reliable to construct POD reduced-order models with a good accuracy. When non-homogeneous boundary conditions have to be taken into account, the best formulation relies on the homogenization of the POMs by means of the control function. However, the LMM (or in the last instance the PM) could also be used if the determination of the control function is complicated or if problems concerning the time derivative of the excitation arise. Finally, the results of the last paragraph show that hybrid POD is a rather robust method: the POMs obtained from snapshots computed for a simple excitation (e.g. the response to an initial condition) seem to provide a good basis to rebuild responses of diverse types, even if the latter are far from the response used to compute the POMs.

This study that fits into the framework of the construction of reduced-order models for coupled fluid-structure systems gives prominence to interesting results concerning the potential of the hybrid POD to 
build an efficient reduced-order model and is therefore encouraging us to use this formulation for future applications, the next one being the reduction of the fluid.

\section{References}

[1] M. Loève, Probability Theory, D. Van Nostrand, Princeton, NJ, 1960.

[2] S. Volkwein, Proper orthogonal decomposition and singular value decomposition, SFB-Preprint No. 153, 1999 /www.uni-graz.at/ imawww/volkwein/svd.ps .

[3] Y. Liang, H. Lee, S. Lim, W. Lin, K. Lee, C. Wu, Proper orthogonal decomposition and its applications - part I: theory, Journal of Sound and Vibration 252 (3) (2002) 527-544.

[4] J. Lumley, The structures of inhomogeneous turbulent flow, in: A.M. Yaglom, V.I. Tatarski (Eds.), Atmospheric Turbulence and Radio Wave Propagation, Nauka, Moscow, 1967, pp. 166-178.

[5] D. Lucia, P. Beran, W. Silva, Reduced-order modeling: new approaches for computational physics, Progress in Aerospace Science 40 (2004) 51-117.

[6] E. Dowell, K. Hall, J. Thomas, R. Florea, B. Epureanu, J. Hegg, Reduced order models in unsteady aerodynamics, AIAA Paper 1999-1261.

[7] G. Kerschen, J.-C. Golinval, A. Vakakis, L. Bergman, The method of proper orthogonal decomposition for dynamical characterization and order reduction of mechanical systems: an overview, Nonlinear Dynamics 41 (2005) 147-169.

[8] B. Feeny, R. Kappagantu, On the physical interpretation of proper orthogonal modes in vibrations, Journal of Sound and Vibration 211 (4) (1998) 607-616.

[9] G. Kerschen, J.-C. Golinval, Physical interpretation of the proper orthogonal modes using the singular value decomposition, Journal of Sound and Vibration 249 (5) (2002) 849-865.

[10] M. Amabili, A. Sarkar, M. Païdoussis, Chaotic vibrations of circular cylindrical shells: Galerkin versus reduced-order models via the proper orthogonal decomposition method, Journal of Sound and Vibration 290 (3-5) (2006) 736-762.

[11] S. Bellizzi, R. Sampaio, POMs analysis of randomly vibrating systems obtained from Karhunen-Loève expansion, Journal of Sound and Vibration 297 (3-5) (2006) 774-793.

[12] M. Azeez, A. Vakakis, Proper orthogonal decomposition (POD) of a class of vibroimpact oscillations, Journal of Sound and Vibration 240 (5) (2001) 859-889.

[13] R. Sampaio, C. Soize, Remarks on the efficiency of POD for model reduction in non-linear dynamics of continuous elastic systems, International Journal for Numerical Methods in Engineering 72 (2007) 22-45.

[14] C. Fletcher, Computational Galerkin Methods, Springer, Berlin, 1984.

[15] K. Tang, W. Graham, J. Peraire, Active flow control using a reduced order model and optimum control, 1996 〈citeseer.ist.psu.edu/ tang96active.html $\rangle$.

[16] P. Holmes, J. Lumley, G. Berkooz, Turbulence, Coherent Structures, Dynamical Systems and Symmetry, Cambridge University Press, Cambridge, 1996.

[17] L. Sirovich, Turbulence and the dynamics of coherent structures parts I-III, Quarterly of Applied Mathematics XLV (1987) 561-590.

[18] Y. Tamura, S. Suganuma, H. Kikuchi, K. Hibi, Proper orthogonal decomposition of random wind pressure field, Journal of Fluids and Structures 13 (7-8) (1999) 1069-1095.

[19] D. Rempfer, On low-dimensional Galerkin models for fluid flow, Theoretical and Computational Fluid Dynamics 14 (2) (2000) 75-88.

[20] K.-J. Bathe, Finite Element Procedures, Prentice-Hall, Englewood Cliffs, NJ, 1996. 